\title{
DEFORMATIONS OF KODAIRA MANIFOLDS
}

\author{
GUEO GRANTCHAROV* \\ Department of Mathematics, Florida International University, Miami, FL 33159, U.S.A. \\ COLIN MCLAUGHLIN ${ }^{\dagger}$ \\ Department of Mathematics, University of California at Riverside, Riverside CA 92521, U.S.A. \\ HENRIK PEDERSEN ${ }^{\ddagger}$ \\ Department of Math. and Comp. Sci., University of Southern Denmark, Campusvej 55, \\ Odense M, DK-5230, Denmark \\ and YAT SUN POON ${ }^{\S}$ \\ Department of Mathematics, University of California at Riverside, Riverside CA 92521, U.S.A.
}

(Received 3 March, 2003; accepted 21 March, 2003)

\begin{abstract}
Using compact simple Lie groups and Heisenberg groups, we combine and generalize the constructions of complex structures on Kodaira surfaces and Hopf surfaces. We identify locally complete parameter spaces of deformations of these spaces and analyze the deformation of Kodaira manifolds in details.

2000 Mathematics Subject Classification. Primary 32G05. Secondary 53C15, $53 \mathrm{C} 56,57 \mathrm{~S} 25,17 \mathrm{~B} 30$.
\end{abstract}

1. Introduction. Kodaira surfaces are elliptic fibrations over elliptic curves with $b_{3}=3[2, \mathbf{1 3}]$. They can be considered as the compact quotient of $\mathbf{C}^{2}$ with respect to a discrete subgroup of the affine group of $\mathbf{C}^{2}$. Taking this point of view, Borcea studied the moduli space of Kodaira surfaces [4]. Alternatively a Kodaira surface is a quotient of a one-dimensional nilpotent extension of the three-dimensional Heisenberg group. In this paper we consider a nilpotent extension $H_{2 n+1} \times \mathbf{R}$ of the $(2 n+1)$-dimensional Heisenberg group $H_{2 n+1}$. We define a left-invariant complex structure on this extension and consider the quotient by a co-compact lattice $\Gamma$. Then we study the moduli of complex structures on these Kodaira manifolds $\Gamma \backslash\left(H_{2 n+1} \times \mathbf{R}\right)$.

Hopf surfaces are complex manifolds diffeomorphic to $S^{1} \times S^{3}$ [2]. A special one is $\mathrm{U}(1) \times \mathrm{SU}(2)$ with left-invariant complex structure. The moduli problem on Hopf surfaces was investigated by several authors including Dabrowski [7]. The investigation of moduli on Hopf surfaces can be extended to $\mathrm{U}(1) \times G$, where $G$ is an odd-dimensional compact simple Lie group. Left-invariant complex structures on these manifolds were studied by Samelson [17]. Using Bott-Borel-Weil theory, the cohomology of the tangent sheaf on such complex manifolds was calculated in [12, Appendix 2].

\footnotetext{
*Partially supported by NSF DMS-0209306 and European contract HPRN-CT-2000-00101.

${ }_{7}^{\ddagger}$ Partially supported by the European contract HPRN-CT-2000-00101.

${ }^{\S}$ Partially supported by NSF DMS-0204002.
} 
We combine the construction of complex structures on Kodaira manifolds with Samelson's construction to define a left-invariant complex structure on $H_{2 n+1} \times G$. Using co-compact subgroups $\Gamma$ in $H_{2 n+1}$, we obtain compact complex manifolds $X=$ $\left(\Gamma \backslash H_{2 n+1}\right) \times G$ with features of both the Kodaira manifold $\left(\Gamma \backslash H_{2 n+1}\right) \times \mathrm{U}(1)$ and the generalized Hopf surface $\mathrm{U}(1) \times G$. We investigate the complex deformations of $X$. One of our main general results is a computation of $H^{1}\left(X, \Theta_{X}\right)$ together with an analysis of integrability. This analysis uses Kuranishi theory and work of Griffiths $[\mathbf{1 5}, \mathbf{1 1}]$. After identifying the cohomology groups, we apply both the Kodaira-Spencer and the Kuranishi method to investigate the integrability of the parameters.

As an application, we analyze the structure of the moduli space of invariant complex structures on Kodaira manifolds in details. To state this result, we shall use $D^{*}$ to denote the punctured unit disc and $\mathcal{H}_{n, k}$ to denote the flag domain $\operatorname{Sp}(2 n, \mathbf{R}) / \mathrm{U}_{n-k, k}(\mathbf{C})$.

THEOREM. The moduli space of invariant complex structures on the complex $2(\ell+1)$ dimensional Kodaira manifold $\Gamma \backslash H_{4 \ell+3} \times S^{1}$ is the topological space

$$
\mathcal{M}=\cup_{j=0}^{\ell}\left(\mathcal{H}_{2 \ell+1,2 \ell+1-4 j} / \operatorname{Sp}(4 \ell+2, \mathbf{Z})\right) \times D^{*} .
$$

The moduli space of invariant complex structures on the complex $2 \ell+1$-dimensional Kodaira manifold $\Gamma \backslash H_{4 \ell+1} \times S^{1}$ is the topological space

$$
\mathcal{M}=\left(\cup_{j=0}^{2 \ell} \mathcal{H}_{2 \ell, 2 \ell-2 j} / \operatorname{Sp}(4 \ell, \mathbf{Z})\right) \times D^{*}
$$

This result may be viewed as a mix of those in [3] on moduli of complex structures of torus via an elliptic fibration over torus. It certainly is a generalization of Borcea's work in complex dimension 2 [4]. In his case, he benefits from classification of Kodaira surfaces and hence the qualification "invariant" is not necessary. For constructions of homogeneous structures on other nilmanifolds see $[\mathbf{1}, \mathbf{1 6}]$.

2. Kodaira manifolds and its generalizations. In this section, we define Kodaira manifolds in such a way that it generalizes Kodaira surfaces. The key element is the Heisenberg group. Afterward, we go on to extend it to consider a Hopf-type generalization of Kodaira manifolds. To prepare our investigation, we also calculate the relevant cohomology groups.

2.1. The Heisenberg group. The underlying manifold of the Heisenberg group $H_{2 n+1}$ is the space $\mathbf{R}^{2 n+1}$. In terms of the coordinates $\left(x_{1}, y_{1}, \ldots, x_{n}, y_{n}, z\right)$ the group operation is given by

$$
(x, y, z) *\left(x^{\prime}, y^{\prime}, z^{\prime}\right)=\left(x+x^{\prime}, y+y^{\prime}, z+z^{\prime}+\frac{1}{2} \sum_{j=1}^{n}\left(x_{j} y_{j}^{\prime}-y_{j} x_{j}^{\prime}\right)\right) .
$$

and satisfies

$$
(x, y, z)^{-1}=(-x,-y,-z)
$$


If we consider $\mathbf{R}^{2 n+1}$ as $\mathbf{C}^{n} \times \mathbf{R}$, in coordinates with $\zeta \in \mathbf{C}^{n}$ and $t \in \mathbf{R}$ the group law looks like

$$
(\zeta, t) *\left(\zeta^{\prime}, t^{\prime}\right)=\left(\zeta+\zeta^{\prime}, t+t^{\prime}+\frac{1}{2} \operatorname{Im}\left(\zeta^{*} \zeta^{\prime}\right)\right)
$$

where $\zeta^{*}$ is the conjugate transpose of $\zeta$. The tangent vectors $\frac{\partial}{\partial x_{j}}, \frac{\partial}{\partial y_{j}}, \frac{\partial}{\partial z}$ at the identity extend to left invariant vector fields

$$
\tilde{X}_{j}=\frac{\partial}{\partial x_{j}}-\frac{1}{2} y_{j} \frac{\partial}{\partial z} \quad \tilde{Y}_{j}=\frac{\partial}{\partial y_{j}}+\frac{1}{2} x_{j} \frac{\partial}{\partial z} \quad \tilde{Z}=\frac{\partial}{\partial z}
$$

respectively. These left invariant vector fields form a basis for the Heisenberg algebra $\mathfrak{h}_{2 n+1}$. The commutator relations of $\mathfrak{h}_{2 n+1}$ are as follows:

$$
\left[\tilde{X}_{j}, \tilde{Y}_{j}\right]=\tilde{Z} \text { for } 1 \leq j \leq n
$$

with all other brackets being equal to 0 . It follows that the Heisenberg algebra is a twostep nilpotent algebra, with the 1-dimensional subspace $\mathfrak{c}$ spanned by $Z$ as its center. It is known that for all nilpotent Lie groups the exponential map is a surjection, and for a simply connected nilpotent group such as the Heisenberg group the exponential mapping will be a diffeomorphism. The algebra and group operations are related by the Campbell-Baker-Hausdorff formula

$$
\exp (X) * \exp (Y)=\exp \left(X+Y+\frac{1}{2}[X, Y]\right) \text { for } X, Y \in \mathfrak{h}_{2 n+1} .
$$

The inverse of the exponential map will be called log, and formula (4) can also be written as

$$
\log (g * h)=\log (g)+\log (h)+\frac{1}{2}[\log (g), \log (h)] .
$$

The log function satisfies the following familiar identity. We skip the proof.

LEMMA $1 . \log \left(g^{n}\right)=n \log (g)$ for every integer $n$ and every $g \in H_{2 n+1}$.

The quotient of the Heisenberg algebra by the center $\mathfrak{c}$ is the Abelian algebra $\mathfrak{t}_{2 n}$. We have an exact sequence

$$
0 \rightarrow \mathfrak{c} \stackrel{\iota}{\rightarrow} \mathfrak{h}_{2 n+1} \stackrel{\phi}{\rightarrow} \mathfrak{t}_{2 n} \rightarrow 0
$$

The center $\mathfrak{c}$, corresponding (under the exponential map) to the central subgroup $C$ of $H_{2 n+1}$, is given $x=y=0$, and $C$ is isomorphic to $(\mathbf{R},+)$. On the group level we have a homomorphism

$$
\phi: H_{2 n+1} \rightarrow\left(\mathbf{R}^{2 n},+\right)
$$

We consider a discrete subgroup $\Gamma$ of $H_{2 n+1}$ generated by elements $g_{i}, 1 \leq i \leq$ $2 n+1$, satisfying the following relations:

$$
\begin{aligned}
g_{2 i-1} g_{2 i} g_{2 i-1}^{-1} g_{2 i}^{-1} & =g_{2 n+1}^{m} \text { for } i=1,2, \ldots, n \\
g_{i} g_{j} g_{i}^{-1} g_{j}^{-1} & =i d \text { for all other combinations }(i, j) \text { with } i<j
\end{aligned}
$$


for some positive integer $m$ and such that the quotient $\Gamma \backslash H_{2 n+1}$ is compact. We will denote the quotient $\Gamma \backslash H_{2 n+1}$ by $\check{H}_{2 n+1}$. For example we could take $g_{i}=e_{i}$ where $\left(e_{1}, \ldots, e_{2 n+1}\right)$ are the standard basis vectors of $\mathbf{R}^{2 n+1}$. The relation then satisfied is $e_{2 i-1} e_{2 i} e_{2 i-1}^{-1} e_{2 i}^{-1}=e_{2 n+1}$. Such a lattice with $m=1$ will be called a primary lattice.

If we denote by $\Gamma_{0}$ the intersection of $\Gamma$ with $C, \Gamma_{0}$ is isomorphic to the additive group $(\mathbf{Z},+)$. The quotient of $C$ by $\Gamma_{0}$ is the one-dimensional torus group $T^{1}$. The image $\phi(\Gamma)$ in $\mathbf{R}^{2 n}$ is isomorphic to $\mathbf{Z}^{2 n}$, and the quotient $\phi(\Gamma) \backslash \phi\left(H_{2 n+1}\right)$ is isomorphic to the 2 -dimensional torus group $T^{2 n}$.

We now prefer to use a new basis of the algebra $\mathfrak{h}_{2 n+1}$ related to the lattice $\Gamma$. Let

$$
\left.X_{j}\right|_{0}=\log \left(g_{2 j-1}\right),\left.\quad Y_{j}\right|_{0}=\log \left(g_{2 j}\right) \text { for } 1 \leq j \leq n, \text { and }\left.Z\right|_{0}=\log \left(g_{2 n+1}\right) .
$$

with the fields $X_{j}, Y_{j}$ and $Z$ defined at other points by left translation. Combining the Campbell-Baker-Hausdorff formula (4) and the relations (8) we find that the algebra of the vector fields generated by $X_{j}, Y_{j}, Z$ is determined by

$$
\left[X_{j}, Y_{j}\right]=m Z
$$

with all other brackets identically 0 . Now we define 1 -forms $\alpha_{j}$ dual to $X_{j}, \beta_{j}$ dual to $Y_{j}$ and $\gamma$ dual to $Z$. The exterior derivatives are given by the Maurer-Cartan equations

$$
d \alpha_{j}=0, \quad d \beta_{j}=0, \quad d \gamma=-m \sum_{j=1}^{n} \alpha_{j} \wedge \beta_{j} .
$$

2.2. Kodaira manifolds. By taking the direct sum of $\mathfrak{h}_{2 n+1}$ with the one dimensional Lie algebra $\mathfrak{t}_{1}$, we extend the exact sequence (6) by a direct sum of $\iota$ with the identity map:

$$
0 \rightarrow \mathfrak{c} \oplus \mathfrak{t}_{1} \stackrel{\iota}{\rightarrow} \mathfrak{h}_{2 n+1} \oplus \mathfrak{t}_{1} \stackrel{\phi}{\rightarrow} \mathfrak{t}_{2 n} \rightarrow 0
$$

The simply connected Lie groups with algebra $\mathfrak{c} \oplus \mathfrak{t}_{1}$ and $\mathfrak{h}_{2 n+1} \oplus \mathfrak{t}_{1}$ are $C \times \mathbf{R}$ and $H_{2 n+1} \times \mathbf{R}$ respectively. We define $\check{\Gamma}$ to be the product of the lattice $\Gamma$ with the integers $\mathbf{Z}$, and $\check{\Gamma}_{0}$ to be the product of $\Gamma_{0}$ with $\mathbf{Z}$. Then the quotient of $H_{2 n+1} \times \mathbf{R}$ by $\check{\Gamma}$ is diffeomorphic to $\breve{H}_{2 n+1} \times U(1)$ and the quotient of the center $C \times \mathbf{R}$ by $\check{\Gamma}_{0}$ is diffeomorphic to the two-dimensional torus $T^{2}$.

Let $t$ be a coordinate for $\mathbf{R}$ so that the vector field $T=\frac{\partial}{\partial t}$ is left invariant. Then $\left(X_{j}, Y_{j}, Z, T\right)$ forms a basis for the algebra $\mathfrak{h}_{2 n+1} \oplus \mathfrak{t}_{1}$. We define an endomorphism $J$ of the algebra by

$$
J X_{j}=Y_{j}, \quad J Y_{j}=-X_{j}, \quad J Z=T, \quad J T=-Z
$$

and extend it from the tangent space at the identity by left translation. This endomorphism defines an almost complex structure on $H_{2 n+1} \times \mathbf{R}$. Since the only non-trivial Lie bracket is of the type $\left[X_{j}, Y_{j}\right]$, it is easy to check that for any left invariant vector fields $X$ and $Y$

$$
[J X, J Y]=[X, Y] .
$$

If a complex structure satisfies (14), it is said to be Abelian. Since the Nijenhuis tensor will be zero in such case, it is integrable. If we define the 1 -form $\chi$ by $\chi=d t$, 
the space of $(1,0)$-forms is spanned by

$$
\omega_{j}=\alpha_{j}+i \beta_{j} \text { for } 1 \leq j \leq n, \quad \omega_{n+1}=\gamma+i \chi .
$$

These forms have exterior derivatives

$$
\begin{aligned}
d \omega_{j} & =0 \text { for } 1 \leq j \leq n, \\
d \omega_{n+1} & =d \gamma=-m \sum_{j=1}^{n} \alpha_{j} \wedge \beta_{j}=-m \frac{i}{2} \sum_{j=1}^{n} \omega_{j} \wedge \bar{\omega}_{j}
\end{aligned}
$$

because $\omega_{j} \wedge \bar{\omega}_{j}=-(2 i) \alpha_{j} \wedge \beta_{j}$. Since $\bar{\partial} \bar{\omega}_{n+1}=0$, the forms $\bar{\omega}_{j}, 1 \leq j \leq n$ are holomorphic type $(0,1)$-forms on $H_{2 n+1} \times \mathbf{R}$.

Since the complex structure is left-invariant, it descends to an integrable complex structure on the quotient space $\breve{H}_{2 n+1} \times U(1)$. We refer to it as a Kodaira manifold. If the lattice is a primary one, the Kodaira manifold is called primary. The projection $\Psi$ from $\breve{H}_{2 n+1} \times U(1)$ onto the torus $T^{2 n}$ given by taking the quotient by the center is a holomorphic map.

2.3. Complex structures on $\check{H} \times G$. The construction in the last section can be substantially generalized in several directions. Let $G$ be an odd-dimensional compact semi-simple Lie group with Lie algebra $\mathfrak{g}$. Let its rank be $2 m-1$. Samelson proves that there is a left-invariant complex structure $J$ on the direct product $T^{1} \times G$ defined as follows [17]. Let $U$ be a maximal torus of $G$. Let $\Delta^{+}$be a choice of positive roots with respect to the Cartan subalgebra $\mathfrak{u}_{\mathbf{C}}$. Let $\mathfrak{g}_{a}$ be the root spaces. Let $\left\{H_{1}, \ldots H_{2 m-1}\right\}$ be a basis for the real Lie algebra $\mathfrak{u}$, let $Z=H_{0}$ be a non-zero vector in $\mathfrak{t}_{1}$, and let $X_{a}$ be a non-zero vector in $\mathfrak{g}_{a}$. Then the $(1,0)$-vectors with respect to $J$ are in the complex linear span of

$$
\left\{H_{2 k}+i H_{2 k+1}, 0 \leq k \leq m-1 ; \quad X_{a}, a \in \Delta^{+}\right\} .
$$

Consider the product space $H_{2 n+1} \times G$. The tangent space to the identity coset is the vector space $\mathfrak{h}_{2 n+1} \oplus \mathfrak{g} \cong \mathfrak{t}_{2 n} \oplus \mathfrak{c} \oplus \mathfrak{g}$. Define an endomorphism $J$ on this space such that on $\mathfrak{t}_{2 n}, J X_{j}=Y_{j}, J Y_{j}=-X_{j}$. On $\mathfrak{c} \oplus \mathfrak{g}$, define $J$ to be Samelson's complex structure on the tangent space of the identity element of $T^{1} \times G$. Through left-translations, this endomorphism is extended to a left-invariant almost complex structure on $H_{2 n+1} \times G$. The almost complex structure descends to an almost complex structure on $\breve{H} \times G$. By construction, this almost complex structure is $G$-invariant.

This almost complex structure is integrable. It is most convenient to verify the integrability on the non-compact space $H_{2 n+1} \times G$ as the almost complex structure is left-invariant on this space. Since $\mathfrak{t}_{2 n}$ and $\mathfrak{c} \oplus \mathfrak{g}$ are both $J$-invariant and they commute in the algebra $\mathfrak{h}_{2 n+1} \oplus \mathfrak{g}=\mathbf{R}^{2 n} \oplus \mathfrak{c} \oplus \mathfrak{g}$, when $N$ is the Nijenhuis tensor for $J$, then $N(X, Y)=0$ for any $X$ in $\mathbf{R}^{2 n}$ and $Y$ in $\mathfrak{c} \oplus \mathfrak{g}$. If both $X$ and $Y$ are in $\mathbf{R}^{2 n}$, then $N(X, Y)=0$ because on this summand, the almost complex structure is Abelian in the sense of (14). If both $X$ and $Y$ are in $\mathfrak{c} \oplus \mathfrak{g}, N(X, Y)=0$ due to the integrability of Samelson's complex structure. It follows that $\check{H} \times G$ has a $G$-invariant complex structure.

In the above definition of the complex structure on $H_{2 n+1} \times G$, the adjoint action of $\mathfrak{c} \oplus \mathfrak{u}$ on $\left(\mathfrak{h}_{2 n+1} \oplus \mathfrak{g}\right)_{\mathbf{C}}$ preserves the type decomposition. Since the complex structure $J$ on $H_{2 n+1} \times G$ is left-invariant, it is also invariant with respect to the right action of 
the group $C \times U$ on $\breve{H} \times G$. The holomorphic quotient of $\breve{H} \times G$ is the product of the complex n-torus $T^{2 n}$ and the Borel's flag manifold $G / U$. We have the holomorphic projection

$$
\Psi: \check{H} \times G \rightarrow T^{2 n} \times G / U .
$$

The fibers are the flows of the right actions generated by the group $C \times U$. Therefore, the algebra of holomorphic vertical vector fields $\mathfrak{v}$ with respect to $\Psi$ is invariant with respect to the left action of $G$.

Denote $\breve{H} \times G$ by $X$ and $T^{2 n} \times G / U$ by $M$, we have the following exact sequence of holomorphic bundles on $X$.

$$
0 \rightarrow \mathcal{O}_{X}(\mathfrak{v}) \rightarrow \Theta_{X} \rightarrow \Psi^{*} \Theta_{M} \rightarrow 0 .
$$

2.4. Computation of cohomology. Use the notation of (15). Let $\chi_{a}$ be the dual 1 -form for the vector $H_{\ell}$ for $1 \leq \ell \leq 2 m-1, \gamma=\chi_{0}$ the dual of $Z$. Then the 1 -forms

$$
\omega_{n+k+1}=\chi_{2 k}+i \chi_{2 k+1}, \text { for } 0 \leq k \leq m-1
$$

are type $(1,0)$-forms.

Let $X_{a}$ and $X_{b}$ be any pair of positive roots. Then $\left[X_{a}, X_{b}\right]=N_{a b} X_{a+b}$ where $N_{a b}=0$ when $a+b$ is not a root. Therefore,

$$
\begin{aligned}
d \omega_{n+k+1}\left(X_{a}, X_{b}\right) & =X_{a} \omega_{n+k+1}\left(X_{b}\right)-X_{b} \omega_{n+k+1}\left(X_{a}\right)-\omega_{n+k+1}\left(\left[X_{a}, X_{b}\right]\right) \\
& =-N_{a b} \omega_{n+k+1}\left(X_{a+b}\right)=0 . \\
d \omega_{n+k+1}\left(X_{a}, H_{\ell}\right) & =X_{a} \omega_{n+k+1}\left(H_{\ell}\right)-H_{\ell} \omega_{n+k+1}\left(X_{a}\right)-\omega_{n+k+1}\left(\left[X_{a}, H_{\ell}\right]\right) \\
& =-a\left(H_{\ell}\right) \omega_{n+k+1}\left(X_{a}\right)=0 \\
d \omega_{n+k+1}\left(H_{\ell}, H_{\hat{\ell}}\right) & =H_{\ell} \omega_{n+k+1}\left(H_{\hat{\ell}}\right)-H_{\hat{\ell}} \omega_{n+k+1}\left(H_{\ell}\right)-\omega_{n+k+1}\left(\left[H_{\ell}, H_{\hat{\ell}}\right]\right)=0 .
\end{aligned}
$$

It follows that $d \omega_{n+k+1}$ does not have any type (2,0)-part. In other words, $\partial \omega_{n+k+1}=0$. Taking complex conjugation, we find that the linear span

$$
\Omega=\left\langle\bar{\omega}_{n+k+1}, 0 \leq k \leq m-1\right\rangle
$$

is the space of vertical holomorphic $(0,1)$-forms on $\check{H} \times G$. This computation works also when $G$ is the group $\mathrm{U}(1)$.

LEMMA 2. Let $\mathcal{O}_{X}$ and $\Theta_{X}$ be the structure sheaf and the tangent sheaf for $X=$ $\breve{H} \times G$, where $G$ is either a compact semi-simple Lie group of odd-dimension or the circle group $T^{1}$. Let $M=T^{2 n} \times G / U$ when $G$ is semi-simple and $M=T^{2 n}$ when $G$ is the circle group. Then for $p \geq 1$, the direct image sheaves are.

$$
R^{p} \Psi_{*} \mathcal{O}_{X}=\wedge^{p} \Omega \otimes \mathcal{O}_{M} ; \quad R^{p} \Psi_{*} \Psi^{*} \Theta_{M}=\wedge^{p} \Omega \otimes \Theta_{M} .
$$

Proof. The second identity is a consequence of the first identity and the projection formula.

To prove the first formula, note that for any $m$ in the manifold $M$,

$$
\left(R^{p} \Psi_{*} \mathcal{O}_{X}\right)_{m}=H^{p}\left(\Psi^{-1}(m), \mathcal{O}_{X}\right) \cong H^{p}\left(C \times U, \mathcal{O}_{X}\right) .
$$


It has constant rank. Therefore, by Grauert's Theorem, the direct image sheaf is locally free. As $\Psi^{-1}(m)$ is isomorphic to a complex torus, for all $p \geq 1$,

$$
H^{p}\left(\Psi^{-1}(m), \mathcal{O}_{X}\right)=\wedge^{p} H^{1}\left(\Psi^{-1}(m), \mathcal{O}_{X}\right)
$$

The vector bundle $R^{p} \Psi_{*} \mathcal{O}_{X}$ is isomorphic to $\wedge^{p} R^{1} \Psi_{*} \mathcal{O}_{X}$. Since the vertical holomorphic $(0,1)$-forms $\bar{\omega}_{n+k+1}$ are non-zero anywhere, and they form a basis for the space of holomorphic $(0,1)$-form on each fiber of the map $\Psi$, they trivialize the bundle $R^{1} \Psi_{*} \mathcal{O}_{X}$. Therefore, we have

$$
R^{p} \Psi_{*} \mathcal{O}_{X} \cong \wedge^{p} R^{1} \Psi_{*} \mathcal{O}_{X} \cong \wedge^{p} \Omega \otimes \mathcal{O}_{M}
$$

The proof is complete.

LEMMA 3. Let $\mathcal{O}$ and $\Theta$ be the structure sheaf and the tangent sheaf for $X=\check{H} \times G$, where $G$ is either a compact semi-simple Lie group with odd-dimension or the circle group. Let $\mathfrak{v}$ be the algebra of vertical holomorphic vector fields with respect to the projection $\Psi$. Let $\mathfrak{t}^{1,0}$ be the $(1,0)$-part of the complexification of $\mathfrak{t}_{2 n}$. Let $\mathfrak{t}^{*(0,1)}$ be the $(0,1)$-forms. Then

$$
\begin{aligned}
H^{k}\left(X, \mathcal{O}_{X}\right) & =\wedge^{k}\left(\Omega \oplus \mathfrak{t}^{*(0,1)}\right) . \\
H^{k}\left(X, \Psi^{*} \Theta_{M}\right) & =\wedge^{k}\left(\Omega \oplus \mathfrak{t}^{*(0,1)}\right) \otimes\left(\mathfrak{t}^{1,0} \oplus \mathfrak{g}_{\mathbf{C}}\right) .
\end{aligned}
$$

Proof. Let us consider the Leray spectral sequence with respect to the $\bar{\partial}$-operator and the holomorphic projection $\Psi$. One has

$$
E_{2}^{p, q}=H^{p}\left(M, R^{q} \Psi_{*} \mathcal{O}_{X}\right), \quad E_{\infty}^{p, q} \Rightarrow H^{p+q}\left(X, \mathcal{O}_{X}\right)
$$

Due to the last proposition, when $q \geq 1$,

$$
\begin{aligned}
E_{2}^{p, q} & =H^{p}\left(M, \wedge^{q} \Omega \otimes \mathcal{O}_{M}\right)=\wedge^{q} \Omega \otimes H^{p}\left(M, \mathcal{O}_{M}\right) \\
& =\wedge^{q} \Omega \otimes H^{p}\left(T^{2 n} \times G / U, \mathcal{O}_{M}\right)=\wedge^{q} \Omega \otimes\left(\oplus_{a+b=p} H^{a}\left(T^{2 n}, \mathcal{O}\right) \otimes H^{b}(G / U, \mathcal{O})\right) .
\end{aligned}
$$

Due to Bott-Borel-Weil theory, $H^{b}(G / U, \mathcal{O})$ vanishes except when $b=0$. Therefore,

$$
E_{2}^{p, q}=\wedge^{q} \Omega \otimes H^{p}\left(T^{2 n}, \mathcal{O}\right)=\wedge^{q} \Omega \otimes \wedge^{p} \mathfrak{t}^{*(0,1)} .
$$

Note that every element in $E_{2}^{p, q}$ is a linear combination of the tensor products of holomorphic $(0, \mathrm{q})$-forms generated by $\Omega$ and holomorphic $(0, \mathrm{p})$-forms lifted from the base. Since these holomorphic forms are globally defined and the differential $d_{2}$ is generated by the $\bar{\partial}$-operator, $d_{2}=0$. It follows that the Leray spectral sequence degenerates on $E_{2}$-level. Therefore,

$$
H^{k}\left(X, \mathcal{O}_{X}\right)=\oplus_{p+q=k} E_{2}^{p, q}=\wedge^{k}\left(\Omega \oplus \mathfrak{t}^{*(0,1)}\right) .
$$

Next, Leray spectral sequence for $\Psi^{*} \Theta_{M}$ gives

$$
E_{2}^{p, q}=H^{p}\left(M, R^{q} \Psi_{*} \Psi^{*} \Theta_{M}\right), \quad E_{\infty}^{p, q} \Rightarrow H^{p+q}\left(X, \Psi^{*} \Theta_{M}\right) .
$$


Due to the last proposition and Kunneth formula, $E_{2}^{p, q}$ is equal to

$$
\begin{aligned}
& H^{p}\left(M, \wedge^{q} \Omega \otimes \Theta_{M}\right)=\wedge^{q} \Omega \otimes H^{p}\left(M, \Theta_{M}\right)=\wedge^{q} \Omega \otimes \oplus_{k=0}^{p}\left(H^{k}\left(T^{2 n}, \Theta_{T^{2 n}}\right)\right. \\
& \left.\otimes H^{p-k}(G / U, \mathcal{O}) \oplus H^{p-k}\left(T^{2 n}, \mathcal{O}\right) \otimes H^{k}\left(G / U, \Theta_{G / U}\right)\right)
\end{aligned}
$$

Due to Bott-Borel-Weil theory, $H^{b}(G / U, \Theta)$ and $H^{b}(G / U, \mathcal{O})$ vanish except when $b=0$ and $H^{0}(G / U, \Theta)=\mathfrak{g}_{\mathbf{C}}$. Therefore,

$$
E_{2}^{p, q}=\wedge^{q} \Omega \otimes \wedge^{p} \mathfrak{t}^{*(0,1)} \otimes\left(\mathfrak{t}^{1,0} \oplus \mathfrak{g}_{\mathbf{C}}\right) .
$$

Note that elements in $\mathfrak{t}^{1,0} \oplus \mathfrak{g}_{\mathbf{C}}$ are holomorphic vector fields on $M$ and hence globally defined sections of $\Psi^{*} \Theta_{M}$ on $X$. Elements in $\wedge^{q} \Omega$ are pulled back to globally defined holomorphic $(0, \mathrm{q})$-forms on $X$. Most important, elements in $\wedge^{p} \mathrm{t}^{*(0,1)}$ are globally defined holomorphic ( $0, \mathrm{p})$-forms on $X$, the operator $d_{2}$ is identically zero. Therefore, the spectral sequence degenerates at $E_{2}$. We have

$$
H^{k}\left(X, \Psi^{*} \Theta_{M}\right)=\oplus_{p+q=k} E_{2}^{p, q}=\wedge^{k}\left(\Omega \oplus \mathfrak{t}^{*(0,1)}\right) \otimes\left(\mathfrak{t}^{1,0} \oplus \mathfrak{g}_{\mathbf{C}}\right) .
$$

The proof is complete.

Now we are able to compute the relevant cohomology. Recall that $\mathfrak{v}$ is the algebra of the vertical holomorphic vector fields with respect to the projection $\Psi$ from $\mathscr{H} \times G$ onto $T^{2 n} \times G / U$. Let $\mathfrak{v}_{0}$ be the complex linear span of $Z-i H_{1}$ in $\mathfrak{v}$. Let $\mathfrak{v}_{1}$ be the complex linear span of $\left\{H_{2 \ell}+i H_{2 \ell+1}: 1 \leq m-1\right\}$. Define $\mathcal{N}=\Omega \otimes \mathfrak{v}_{0} \oplus \mathfrak{t}^{*(0,1)} \odot \mathfrak{t}^{1,0}$ and $\mathcal{S}=H^{1}\left(X, \mathcal{O}_{X}\right) \otimes\left(\mathfrak{v}_{1} \oplus \mathfrak{g}_{\mathbf{C}}\right)=\left(\Omega \oplus \mathfrak{t}^{*(0,1)}\right) \otimes\left(\mathfrak{v}_{1} \oplus \mathfrak{g}_{\mathbf{C}}\right)$.

Proposition 1. Let $\mathcal{O}_{X}, \Theta_{X}$ be the structure sheaf and the tangent sheaf for $X=\check{H} \times$ $G$, where $G$ is a compact semi-simple Lie group of odd-dimension. Then $H^{0}\left(X, \Theta_{X}\right)=$ $\mathfrak{v} \oplus \mathfrak{g}_{\mathbf{C}}$ and $H^{1}\left(X, \Theta_{X}\right)=\mathcal{N} \oplus \mathcal{S}$.

Proof. The induced long exact sequence of (17) yields

$$
\rightarrow H^{k}(X, \mathcal{O}) \otimes \mathfrak{v} \rightarrow H^{k}\left(X, \Theta_{X}\right) \rightarrow H^{k}\left(X, \Psi^{*} \Theta_{M}\right) \stackrel{\delta_{k}}{\rightarrow} H^{k+1}(X, \mathcal{O}) \otimes \mathfrak{v} \rightarrow .
$$

By the last proposition, the coboundary map is

$$
\delta_{k}: \wedge^{k}\left(\Omega \oplus \mathfrak{t}^{*(0,1)}\right) \otimes\left(\mathfrak{t}^{1,0} \oplus \mathfrak{g}_{\mathbf{C}}\right) \rightarrow \wedge^{k+1}\left(\Omega \oplus \mathfrak{t}^{*(0,1)}\right) \otimes \mathfrak{v} .
$$

Now we have to chase the diagram to calculate the coboundary maps. To do so, we choose a metric $h$ on $\breve{H} \times G$ satisfying the following conditions: the restriction of $h$ onto $\breve{H}$ is the Euclidean metric such that the coordinate vector fields are orthonormal. The restriction onto $G$ is the bi-invariant metric such that $h\left(H_{1}, H_{1}\right)=h(Z, Z)=1$. We also assume that the basis $\left\{H_{1}, \ldots, H_{2 m-1}\right\}$ is orthonormal with respect to $h$. It is not hard to see that this is a Hermitian metric on $\breve{H} \times G$. Let $\nabla$ be the Chern connection on $\Theta_{X}$. We use the $\bar{\partial}$-operator twisted by the Chern connection to construct the cohomology for $\Theta_{X}$.

There are two types of elements in $H^{k}\left(X, \Psi^{*} \Theta_{M}\right)$. One type is given by $\Upsilon \otimes V$ where $\Upsilon$ is a holomorphic (0,k)-form contained in $\wedge^{k}\left(\Omega \oplus \mathrm{t}^{*(0,1)}\right)$ and $V$ is an element in $\mathfrak{g}_{\mathrm{C}}$. Since $V$ has a natural holomorphic lifting to $X$ and $\Upsilon$ is a holomorphic k-form on $X, \Upsilon \otimes V$ is a holomorphic $(0, \mathrm{k})$-form on $X$ with values in $\Theta_{X}$. As the Chern 
connection is compatible with the holomorphic structure, $\bar{\partial}_{\nabla}(\Upsilon \otimes V)=0$. Therefore, $\delta_{k}(\Upsilon \otimes V)=0$.

Another type is given by $\Upsilon \otimes\left(\frac{\partial}{\partial x_{j}}-i \frac{\partial}{\partial y_{j}}\right)$. Its lifting is $\Upsilon \otimes\left(X_{j}-i Y_{j}\right)$. The holomorphic vector field $\frac{\partial}{\partial x_{j}}-i \frac{\partial}{\partial y_{j}}$ on $T^{2 n} \times G / U$ can also be lifted to $X_{j}-i Y_{j}$. Since the vector field $X_{j}$ is obtained by left-translation, its flow is generated by right multiplication. Therefore, it is not necessarily a holomorphic vector field. To calculate the coboundary map, we recall an observation of Gauduchon [9] that $\bar{\partial}_{A}^{\nabla} B=[A, B]^{1,0}$ when $A$ is a $(0,1)$-vector and $B$ is a $(1,0)$-vector field. Keeping in mind that $X_{j}-i Y_{j}$ is a vector field along the first factor of the product $\breve{H} \times G$, we have

$$
\begin{aligned}
& \delta_{0}\left(\frac{\partial}{\partial x_{j}}-i \frac{\partial}{\partial y_{j}}\right)=\bar{\partial}^{\nabla}\left(X_{j}-i Y_{j}\right) \\
& \quad=\frac{1}{2}\left(\sum_{k=1}^{n}\left[X_{k}+i Y_{k}, X_{j}-i Y_{j}\right]^{1,0} \otimes \bar{\omega}_{k}+\left[Z+i H_{1}, X_{j}-i Y_{j}\right]^{1,0} \otimes \bar{\omega}_{n+1}\right) \\
& \quad=-i \delta_{j k} Z^{1,0} \otimes \bar{\omega}_{k}=-\frac{1}{2} i\left(Z-i H_{1}\right) \otimes \bar{\omega}_{j} .
\end{aligned}
$$

In particular, kernel of $\delta_{0}$ is $\mathfrak{g}_{\mathbf{C}}$. As $\mathfrak{v}_{0}$ is the complex linear span of $Z-i H_{1}$ in $\mathfrak{v}$, the image of $\delta_{0}$ is $\mathfrak{v}_{0} \otimes \mathfrak{t}^{*(0,1)}$. Therefore, we have $H^{0}(X, \Theta)=\mathfrak{v} \oplus \mathfrak{g}_{\mathbf{C}}$.

To calculate $\delta_{1}$, note that $\left\{\omega_{j}: 1 \leq j \leq m+n\right\}$ is a basis for $\mathrm{t}^{*(0,1)} \oplus \Omega$. Since they are holomorphic and elements in $\mathfrak{g}_{\mathbf{C}}$ has a natural holomorphic lifting, $\left(\Omega \oplus \mathfrak{t}^{*(0,1)}\right) \otimes \mathfrak{g}_{\mathbf{C}}$ is in the kernel of $\delta_{1}$. On the other hand, for $0 \leq k \leq m$ and $1 \leq j \leq n$,

$$
\bar{\partial}^{\nabla}\left(\bar{\omega}_{n+k} \otimes\left(X_{j}-i Y_{j}\right)\right)=\bar{\omega}_{n+k} \wedge \bar{\partial}^{\nabla}\left(X_{j}-i Y_{j}\right)=2 i \bar{\omega}_{n+k} \wedge \bar{\omega}_{j} \otimes\left(Z-i H_{1}\right) .
$$

Therefore, the restriction of $\delta_{1}$ on $\Omega \otimes \mathfrak{t}^{1,0}$ is an injective map. Its image is $\Omega \otimes \mathfrak{t}^{*(0,1)} \otimes$ $\mathfrak{v}_{0}$.

Similarly, for $0 \leq k, j \leq n$,

$$
\bar{\partial}^{\nabla}\left(\bar{\omega}_{k} \otimes\left(X_{j}-i Y_{j}\right)\right)=\bar{\omega}_{k} \wedge \bar{\partial}^{\nabla}\left(X_{j}-i Y_{j}\right)=2 i \bar{\omega}_{k} \wedge \bar{\omega}_{j} \otimes\left(Z-i H_{1}\right) .
$$

Therefore, the kernel of the restriction of $\delta_{1}$ on $\mathfrak{t}^{*(0,1)} \otimes \mathfrak{t}^{1,0}$ is the symmetric product $\mathfrak{t}^{*(0,1)} \odot \mathfrak{t}^{1,0}$. The image is $\wedge^{2} \mathfrak{t}^{*(0,1)} \otimes \mathfrak{v}_{0}$.

By definition of $\mathfrak{v}_{1}$, the following sequence is exact.

$$
0 \rightarrow \Omega \otimes \mathfrak{v} \oplus \mathfrak{t}^{*(0,1)} \otimes \mathfrak{v}_{1} \rightarrow H^{1}(X, \Theta) \rightarrow\left(\Omega \oplus \mathfrak{t}^{*(0,1)}\right) \otimes \mathfrak{g}_{\mathbf{C}} \oplus \mathfrak{t}^{*(0,1)} \odot \mathfrak{t}^{1,0} \rightarrow 0 .
$$

This completes the proof.

In the above description of holomorphic vector fields, we find a direct sum decomposition. In fact, this is a direct sum decomposition of Lie algebras. Any element in $\mathfrak{g}_{\mathrm{C}}$ is represented by $V=C-i J C$ where $C$ is a right-invariant real vector field on $G$. Since $C$ is holomorphic, $L_{C} J=0$. Since right multiplications commute with left multiplications, it is clear that the vector field $C$ commutes with the left-invariant vector fields $\left\{H_{0}, H_{1}, \ldots, H_{2 m-1}\right\}$ where $H_{0}=Z$. We note that these left-invariant vector fields are holomorphic. i.e. $L_{H_{\ell}} J=0$. Therefore,

$$
\left[H_{\ell}, J C\right]=J\left[H_{\ell}, C\right]=0 .
$$


Since $\mathfrak{v}$ is generated by an Abelian group,

$$
\left[\mathfrak{v}, \mathfrak{g}_{\mathbf{C}}\right]=0, \quad \text { and } \quad[\mathfrak{v}, \mathfrak{v}]=0 .
$$

We shall refer to the component $\mathfrak{v}$ as the nilpotent part and to $\mathfrak{g}_{\mathbf{C}}$ as the semi-simple part of the algebra of holomorphic vector fields on $X$.

In the case when $G=\mathrm{U}(1)$, the semi-simple part is equal to zero. The above proof yields the following.

Proposition 2. Let $\mathcal{O}_{X}, \Theta_{X}$ be the structure sheaf and the tangent sheaf for $X=$ $\check{H} \times \mathrm{U}(1)$. Let $\mathfrak{v}$ be the algebra of vertical holomorphic vector fields with respect to the projection $\Psi$ from $\check{H} \times \mathrm{U}(1)$ onto $T^{2 n}$. Let $\mathfrak{t}^{1,0}$ be the $(1,0)$-part of the complexification of $\mathfrak{t}_{2 n}$. Let $\mathrm{t}^{*(0,1)}$ be the $(0,1)$-forms. Then

$$
\begin{aligned}
& H^{0}\left(X, \Theta_{X}\right)=\mathfrak{v} \cong \mathbf{C} . \\
& H^{1}\left(X, \Theta_{X}\right)=\mathfrak{t}^{*(0,1)} \odot \mathfrak{t}^{1,0} \oplus \Omega \otimes \mathfrak{v}_{0} \cong \mathbf{C}^{\frac{1}{2} n(n+1)} \oplus \mathbf{C} .
\end{aligned}
$$

3. Kodaira-Spencer-Kuranishi theory. The computations in the last section have identified the virtual parameter space of deformations. Since $H^{2}\left(X, \Theta_{X}\right)$ does not vanish, we need to investigate the obstructions in some depth before we can identify the directions in which the virtual parameters of deformations are integrable.

3.1. The Nijenhuis bracket. If $\bar{\omega} \otimes V$ is a vector-valued $(0,1)$-form representing an element in $H^{1}\left(X, \Theta_{X}\right)$, the obstruction for it to be the tangent of a one-parameter deformation is contained in a bracket operation:

$$
\{\cdot, \cdot\}: H^{1}\left(X, \Theta_{X}\right) \times H^{1}\left(X, \Theta_{X}\right) \rightarrow H^{2}\left(X, \Theta_{X}\right) .
$$

We call this bracket the Nijenhuis bracket $[\mathbf{8}, \mathbf{1 0}]$. This obstruction was discussed by Kodaira-Spencer in [14, Section 6] and it plays a critical role in Kuranishi theory [15]. It is defined as follows. When $\Omega_{1}$ and $\Omega_{2}$ are vector-valued 1-forms representing elements in $H^{1}\left(X, \Theta_{X}\right)$, and when $A$ and $B$ are $(0,1)$-vector fields, then

$$
\begin{aligned}
\left\{\Omega_{1}, \Omega_{2}\right\}(A, B) \\
=\left[\Omega_{1}(A), \Omega_{2}(B)\right]-\left[\Omega_{1}(B), \Omega_{2}(A)\right] \\
\quad+\Omega_{1}\left(-\left[A, \Omega_{2}(B)\right]+\left[B, \Omega_{2}(A)\right]\right)+\Omega_{2}\left(-\left[A, \Omega_{1}(B)\right]+\left[B, \Omega_{1}(A)\right]\right) .
\end{aligned}
$$

In the case when $\Omega_{1}=\bar{\omega} \otimes V$ and $\Omega_{2}=\bar{\omega}^{\prime} \otimes V^{\prime}$, by Griffiths [11, Proposition 10.5] the Dolbeault representative for $\left\{\bar{\omega} \otimes V, \bar{\omega}^{\prime} \otimes V^{\prime}\right\}$ is

$$
\bar{\omega}^{\prime} \wedge L_{V^{\prime}} \bar{\omega} \otimes V+\bar{\omega} \wedge L_{V} \bar{\omega}^{\prime} \otimes V^{\prime}+\bar{\omega} \wedge \bar{\omega}^{\prime} \otimes\left[V, V^{\prime}\right]
$$

LeMMA 4. Suppose $X$ is the complex manifold $\breve{H} \times G$ where $G$ is a compact semisimple Lie group. The Nijenhuis bracket between any pair of elements of $\mathcal{N} \oplus \mathcal{S}$ is equal to zero except possibly when they both are in $\left(\Omega \oplus \mathfrak{t}^{*(0,1)}\right) \otimes \mathfrak{g}_{\mathbf{C}}$. When $\bar{\omega} \otimes V$ and $\bar{\omega}^{\prime} \otimes V^{\prime}$ are both in $\left(\Omega \oplus \mathfrak{t}^{*(0,1)}\right) \otimes \mathfrak{g}_{\mathbf{C}}$, then

$$
\left\{\bar{\omega} \otimes V, \bar{\omega}^{\prime} \otimes V^{\prime}\right\}=\bar{\omega} \wedge \bar{\omega}^{\prime} \otimes\left[V, V^{\prime}\right] .
$$


Proof. The bundle of $(0,1)$-vectors on $\check{H} \times G$ is globally and smoothly trivialized by

$$
X_{j}+i Y_{j}, 1 \leq j \leq n, \quad H_{2 \ell}+i H_{2 \ell+1}, 0 \leq \ell \leq m-1, \quad \mathfrak{g}_{-}=\left\{\bar{W}_{1}, \ldots, \bar{W}_{N}\right\},
$$

where $\mathfrak{g}_{-}$is the complex vector space of negative roots for $\mathfrak{g}_{\mathbf{C}}$ with respect to the chosen maximal torus $U$ and the vectors $W_{j}$ form a basis. Let us assume that $A$ and $B$ are elements in the basis of the above trivialization.

If $\Omega_{1}$ and $\Omega_{2}$ are both in $\mathfrak{t}^{*(0,1)} \odot \mathfrak{t}^{1,0}$, then $\left[\Omega_{1}(A), \Omega_{2}(B)\right]=0$ for all $A, B$. Also, $\left[A, \Omega_{i}(B)\right]$ is in the linear span of $Z$ and hence is in the kernel of $\Omega_{j}$. Therefore, $\left\{\mathfrak{t}^{*(0,1)} \odot \mathfrak{t}^{1,0}, \mathfrak{t}^{*(0,1)} \odot \mathfrak{t}^{1,0}\right\}=0$.

If $\Omega_{1}$ and $\Omega_{2}$ are elements contained in $\mathfrak{t}^{*(0,1)} \otimes \mathfrak{v}_{1} \oplus \Omega \otimes \mathfrak{v}_{0} \oplus \Omega \otimes \mathfrak{v}_{1}$, then $\left[\Omega_{1}(A), \Omega_{2}(B)\right]=0$ for any $A$ and $B$. For $i, j=1,2, \Omega_{i}(A)$ is in $\mathfrak{v},\left[B, \Omega_{i}(A)\right]$ is contained in $\mathfrak{g}_{-}$which is in the kernel of $\Omega_{j}$. Therefore,

$$
\left\{\mathfrak{t}^{*(0,1)} \otimes \mathfrak{v}_{1} \oplus \Omega \otimes \mathfrak{v}_{0} \oplus \Omega \otimes \mathfrak{v}_{1}, \mathfrak{t}^{*(0,1)} \otimes \mathfrak{v}_{1} \oplus \Omega \otimes \mathfrak{v}_{0} \oplus \Omega \otimes \mathfrak{v}_{1}\right\}=0 .
$$

A similar computation shows that $\left\{\mathfrak{t}^{*(0,1)} \otimes \mathfrak{v}_{1}, \mathfrak{t}^{*(0,1)} \odot \mathfrak{t}^{1,0}\right\}=0$.

In the case when $\Omega_{1}=\bar{\omega}_{n+k} \otimes\left(H_{2 \ell}-i H_{2 \ell+1}\right)$ is in $\Omega \otimes\left(\mathfrak{v}_{0} \oplus \mathfrak{v}_{1}\right)$, and $\Omega_{2}=\bar{\omega}_{i} \otimes$ $\left(X_{j}-i Y_{j}\right)+\bar{\omega}_{j} \otimes\left(X_{i}-i Y_{i}\right)$ is in $\mathfrak{t}^{*(0,1)} \odot \mathfrak{t}^{1,0}$, we note that the range of the adjoint action of $H_{\ell}$ 's are contained in the kernel of $\bar{\omega}_{i}$ and $\bar{\omega}_{j}$. Therefore, $\left[\Omega_{1}, \Omega_{2}\right](A, B)$ could be non-zero only when $A=X_{m}+i Y_{m}$ and $B=X_{l}+i Y_{l}$ for some integers $1 \leq m, l \leq n$. In this case,

$$
\begin{aligned}
\left\{\Omega_{1},\right. & \left.\Omega_{2}\right\}(A, B) \\
= & -\bar{\omega}_{n+k}\left(\left[X_{m}+i Y_{m}, \delta_{i l}\left(X_{j}-i Y_{j}\right)+\delta_{j l}\left(X_{i}-i Y_{i}\right)\right]\right. \\
& \left.-\left[X_{l}+i Y_{l}, \delta_{i m}\left(X_{j}-i Y_{j}\right)+\delta_{j m}\left(X_{i}-i Y_{i}\right)\right]\right)\left(H_{2 \ell}-i H_{2 \ell+1}\right) \\
= & -8 i \bar{\omega}_{n+k}(Z)\left(\delta_{i l} \delta_{m j}+\delta_{j l} \delta_{m i}-\delta_{i m} \delta_{l j}-\delta_{j m} \delta_{l i}\right)=0 .
\end{aligned}
$$

It follows that $\left\{\Omega \otimes\left(\mathfrak{v}_{0} \oplus \mathfrak{v}_{1}\right), \mathfrak{t}^{*(0,1)} \odot \mathfrak{t}^{1,0}\right\}=0$.

Next, we compute the Nijenhuis bracket involving the holomorphic vector fields in $\mathfrak{g}_{\mathbf{C}}$.

When $\Omega_{1}$ is in $\mathfrak{t}^{*(0,1)} \odot \mathfrak{t}^{1,0}$ and $\Omega_{2}$ is in $\left(\Omega \oplus \mathfrak{t}^{*(0,1)}\right) \otimes \mathfrak{g}_{\mathbf{C}},\left[\Omega_{1}(A), \Omega_{2}(B)\right]=0$ for all $A, B$. Moreover, $\left[A, \Omega_{2}(B)\right]$ is a tangent vector field along $G$. It is in the kernel of $\Omega_{1}$. $\left[A, \Omega_{1}(B)\right]$ is contained in the center of the Heisenberg space. Therefore,

$$
\left\{\Omega_{1}, \Omega_{2}\right\}(A, B)=-\Omega_{2}\left(\left[A, \Omega_{1}(B)\right]-\left[B, \Omega_{1}(A)\right]\right) .
$$

And it could be non-zero only when $A=X_{m}+i Y_{m}, B=X_{l}+i Y_{l}$ for some $m$ and $l$. In such case, a computation similar to (39) shows that $\left\{\mathfrak{t}^{*(0,1)} \odot \mathfrak{t}^{1,0},\left(\Omega \oplus \mathfrak{t}^{*(0,1)}\right) \otimes \mathfrak{g}_{\mathbf{C}}\right\}=$ 0 .

Now if $\Omega_{1}$ is in $\mathfrak{t}^{*(0,1)} \otimes \mathfrak{v}_{1}$ and $\Omega_{2}$ is in $\left(\Omega \oplus \mathfrak{t}^{*(0,1)}\right) \otimes \mathfrak{g}_{\mathbf{C}},\left[\Omega_{1}(A), \Omega_{2}(B)\right]=0$ for all $A, B$. Moreover, $\left[A, \Omega_{1}(B)\right]$ is contained in $\mathfrak{g}_{-}$. It follows that it is contained in the kernel of $\Omega_{2}$. $\left[A, \Omega_{2}(B)\right]$ is a tangent vector field along $G$. It is in the kernel of $\Omega_{1}$. Therefore, $\left\{\mathfrak{t}^{*(0,1)} \otimes \mathfrak{v}_{1},\left(\Omega \oplus \mathfrak{t}^{*(0,1)}\right) \otimes \mathfrak{g}_{\mathbf{C}}\right\}=0$.

To make further computation, we claim that for all $A$ in the basis of the trivialization and for all $V=R-i J R$ where $R$ is right-invariant vector field

$$
\bar{\omega}([A, V])=0
$$


for all $\bar{\omega}$ in $\Omega \oplus \mathfrak{t}^{*(0,1)}$. Since $[A, V]$ is a tangent vector field along $G$, it is clear that it is in the kernel of $\bar{\omega}$ if $\bar{\omega}$ is in $\mathrm{t}^{*(0,1)}$. Since $\left\{H_{0}, H_{1}, \ldots, H_{2 m-1}\right\}$ is a set of orthonormal vectors with respect to the bi-invariant metric $h$, the dual 1 -forms $\chi_{\ell}$ are determined by $\chi_{\ell}(B)=h\left(B, H_{\ell}\right)$.

Let $A$ be any real left-invariant vector field. Let $B=J A$, then $B$ is again a leftinvariant vector field. $A+i B$ is a $(0,1)$-vector field. Since $R$ is right-invariant $[A+$ $i B, R]=0$. Then

$$
\begin{aligned}
& \bar{\omega}_{n+k+1}([A+i B, V]) \\
& \quad=h\left(H_{2 k}-i H_{2 k+1},[A+i B, R-i J R]\right) \\
& \quad=i h\left(H_{2 k}-i H_{2 k+1},[A+i B, J R]\right) \\
& \quad=h\left(H_{2 k},[B, J R]\right)+h\left(H_{2 k+1},[A, J R]\right)+i h\left(H_{2 k},[A, J R]\right)-i h\left(H_{2 k+1},[B, J R]\right) \\
& \quad=-h\left(\left[B, H_{2 k}\right], J R\right)-h\left(\left[A, H_{2 k+1}\right], J R\right)-i h\left(\left[A, H_{2 k}\right], J R\right)+i h\left(\left[B, H_{2 k+1}\right], J R\right) \\
& \quad=-h\left(B,\left[H_{2 k}, J R\right]\right)-h\left(A,\left[H_{2 k+1}, J R\right]\right)-i h\left(A,\left[H_{2 k}, J R\right]\right)+i h\left(B,\left[H_{2 k+1}, J R\right]\right) \\
& \quad=-h\left(B, J\left[H_{2 k}, R\right]\right)-h\left(A, J\left[H_{2 k+1}, R\right]\right)-i h\left(A, J\left[H_{2 k}, R\right]\right)+i h\left(B, J\left[H_{2 k+1}, R\right]\right) \\
& \quad=0 .
\end{aligned}
$$

Therefore, $(41)$ is proved.

Now if $\Omega_{1}$ is in $\Omega \otimes\left(\mathfrak{v}_{0} \oplus \mathfrak{v}_{1}\right)$ and $\Omega_{2}$ is in $\left(\Omega \oplus \mathfrak{t}^{*(0,1)}\right) \otimes \mathfrak{g}_{\mathbf{C}}$, then $\left[\Omega_{1}(A), \Omega_{2}(B)\right]=$ 0 for all $A, B$. Moreover, $\left[A, \Omega_{1}(B)\right] \in \mathfrak{g}_{-}$and $\mathfrak{g}_{-}$is in the kernel of $\Omega_{2}$. Therefore, if $\Omega_{2}=\bar{\omega}_{j} \otimes V$, then with (41),

$$
\left\{\Omega_{1}, \Omega_{2}\right\}(A, B)=-\Omega_{1}\left(\bar{\omega}_{j}(B)[A, V]-\bar{\omega}_{j}(A)[B, V]\right)=0
$$

for $1 \leq j \leq m$. Therefore, $\left\{\Omega \otimes\left(\mathfrak{v}_{0} \oplus \mathfrak{v}_{1}\right),\left(\Omega \oplus \mathfrak{t}^{*(0,1)}\right) \otimes \mathfrak{g}_{\mathbf{C}}\right\}=0$.

Finally, when both $\Omega_{1}=\bar{\omega}_{i} \otimes V_{1}$ and $\Omega_{2}=\bar{\omega}_{j} \otimes V_{2}$ are in $\left(\Omega \oplus \mathfrak{t}^{*(0,1)}\right) \otimes \mathfrak{g}_{\mathbf{C}}$, then equation (41) implies that $\bar{\omega}_{i}\left(\left[A, \bar{\omega}_{j}(B)\right]\right)=0$ for all $A$ and $B$ and $i, j=1,2$. Therefore,

$$
\begin{aligned}
\left\{\Omega_{1}, \Omega_{2}\right\}(A, B) & =\bar{\omega}_{i}(A) \bar{\omega}_{j}(B)\left[V_{1}, V_{2}\right]-\bar{\omega}_{i}(B) \bar{\omega}_{j}(A)\left[V_{1}, V_{2}\right] \\
& =\bar{\omega}_{i} \wedge \bar{\omega}_{j} \otimes\left[V_{1}, V_{2}\right]
\end{aligned}
$$

completing the proof.

Knowing the Nijenhuis bracket among elements in $H^{1}\left(X, \Theta_{X}\right)$, we shall investigate the obstructions to deformations from both Griffiths' approach [11] and from Kuranishi's approach [15].

3.2. Kodaira-Spencer theory. Recall a very useful definition from [11].

Definition 1. Let $\mathcal{A}$ be a subalgebra of $H^{0}\left(X, \Theta_{X}\right)$. The complex manifold $X$ is said to satisfy Condition $D$ with respect to $\mathcal{A}$ if the following hold:

(i) $j: H^{1}\left(X, \mathcal{O}_{X}\right) \otimes \mathcal{A} \rightarrow H^{1}\left(X, \Theta_{X}\right)$ is injective;

(ii) If $V \in \mathcal{A}$ and $\bar{\omega} \in H^{1}\left(X, \mathcal{O}_{X}\right)$, then there exists a $C^{\infty}$ function $f=f(V, \bar{\omega})$ such that $L_{V} \bar{\omega}=\bar{\partial} f$.

Let $\mathcal{A}:=\mathfrak{v}_{1} \oplus \mathfrak{g}_{\mathbf{C}}$. Due to (32), $\mathcal{A}$ is a subalgebra. Since $H^{1}\left(X, \mathcal{O}_{X}\right)=\Omega \oplus \mathfrak{t}^{*(0,1)}$, it is also apparent that the tensor product map $j$ is an inclusion. We now have

$$
H^{1}\left(X, \Theta_{X}\right)=\mathcal{N} \oplus \mathcal{S}=\mathcal{N} \oplus H^{1}\left(X, \mathcal{O}_{X}\right) \otimes \mathcal{A}
$$


Lemma 5. The complex manifold $X$ satisfies Condition $D$ with respect to the subalgebra $\mathcal{A}=\mathfrak{v}_{1} \oplus \mathfrak{g}_{\mathbf{C}}$.

Proof. It suffices to verify the second condition in the definition of Condition D.

Suppose that $V$ is in $\mathfrak{v}_{1}$. If $\bar{\omega}$ is in $\mathfrak{t}^{*(0,1)}$, it is clear that $L_{V} \bar{\omega}=0$ because $V$ is tangential to the second factor of $\breve{H} \times G$ and $\mathfrak{t}^{*(0,1)}$ contains forms dual to vectors along the first factor. If $\bar{\omega}$ is in $\Omega$, then $L_{V} \bar{\omega}=0$ because for any left-invariant vector field $A,[V, A]$ is in $\mathfrak{g}_{+} \oplus \mathfrak{g}_{-}$which is in the kernel of $\bar{\omega}$.

Suppose that $V$ is in $\mathfrak{g}_{\mathbf{C}}$. Let $V=R-i J R$ where $R$ is a right invariant vector field. If $\bar{\omega}$ is in $\mathrm{t}^{*(0,1)}$, it is clear that $L_{V} \bar{\omega}=0$ because $V$ is tangential to the second factor of $\breve{H} \times G$ and $\mathrm{t}^{*(0,1)}$ contains forms dual to vectors along the first factor.

Suppose that $\bar{\omega}$ is in $\Omega$. In particular, this is a left-invariant holomorphic $(0,1)$-form and $d \bar{\omega}$ is a type $(1,1)$-form.

Treating $\check{H} \times G$ as a principal $C \times U$-bundle over $M=T^{2 n} \times G / U$, we consider $\left(\bar{\omega}_{n+1}, \ldots, \bar{\omega}_{n+m}\right)$ as a connection 1-form. In particular, $\left(d \bar{\omega}_{n+1}, \ldots, d \bar{\omega}_{n+m}\right)$ is the curvature form. Note that $d \bar{\omega}_{n+1}=\sum_{j=1}^{n} \omega_{j} \wedge \bar{\omega}_{j}-i d \chi_{1}$. When $\bar{\omega}$ is in $\Omega$, it is in the complex linear span of $\left(\bar{\omega}_{n+1}, \ldots, \bar{\omega}_{n+m}\right)$. Therefore, there exists a 2 -form $\eta$ on $G / U$ and a constant $c$ such that $d \bar{\omega}=\Psi^{*}\left(c \sum_{j=1}^{n} \omega_{j} \wedge \bar{\omega}_{j}+\eta\right)$. As the connection is left-invariant with respect to $G$, the curvature form $\eta$ is left-invariant form with respect to $G$.

Since $R$ is a right-invariant vector field, its flow is generated by left-multiplication. As $\eta$ is a left-invariant form, $L_{R} \eta=0$. As $c \sum_{j=1}^{n} \omega_{j} \wedge \bar{\omega}_{j}+\eta$ is a curvature form, $d\left(c \sum_{j=1}^{n} \omega_{j} \wedge \bar{\omega}_{j}+\eta\right)=0$. Yet $d \sum_{j=1}^{n} \omega_{j} \wedge \bar{\omega}_{j}=0$, it follows that $d \iota_{R} \eta=0$. Therefore, $\iota_{R} \eta$ is a DeRham closed 1-form on $G / U$. By Bott-Borel-Weil theory [5], $H^{1}(G / U, \mathbf{C})$ vanishes. Therefore, there exists a function $F$ on $G / U$ such that $\iota_{V} \eta=d F$. Let $f=$ $F \circ \Psi$ be the pull-back of $F$ to $\check{H} \times G$. Then $\iota_{R} d \bar{\omega}=\iota_{R} \Psi^{*} \eta=\Psi^{*} \iota_{R} \eta=\Psi^{*} d F=d f$. Since $\bar{\partial} \bar{\omega}=0$, it follows from type decomposition that $\iota_{V} d \bar{\omega}=\iota_{R-i I R} d \bar{\omega}=\bar{\partial} f$.

As $V$ is a type $(1,0)$-vector, we have $L_{V} \bar{\omega}=d \iota_{V} \bar{\omega}+\iota_{V} d \bar{\omega}=\iota_{V} d \bar{\omega}=\bar{\partial} f$. This completes the proof of the lemma.

Let $\mathfrak{k}_{\mathbf{C}}$ be any choice of Cartan subalgebra in $\mathfrak{g}_{\mathbf{C}}$, then $\mathfrak{v}_{1} \oplus \mathfrak{k}_{\mathbf{C}}$ is a maximal Abelian subalgebra of $\mathcal{A}=\mathfrak{v}_{1} \oplus \mathfrak{g}_{\mathbf{C}}$. As a consequence of Griffiths' result [11, Theorem 12], we have the following result about integrability even if the Nijenhuis bracket is not vanishing.

Proposition 3. Every element of the form $\bar{\omega} \otimes V$ in $H^{1}\left(X, \mathcal{O}_{X}\right) \otimes \mathcal{A}$ is tangent to a 1-parameter family of deformations. For any choice of Cartan subalgebra $\mathfrak{k}_{\mathbf{C}}$ in $\mathfrak{g}_{\mathbf{C}}$, $\left(\Omega \oplus \mathfrak{t}^{*(0,1)}\right) \otimes\left(\mathfrak{v}_{1} \oplus \mathfrak{k}_{\mathbf{C}}\right)$ parametrizes a local deformation space, which is maximal in $H^{1}\left(X, \mathcal{O}_{X}\right) \otimes \mathcal{U}$.

3.3. Kuranishi theory. Next we apply Kuranishi Theory to analyze the summand $\mathcal{N}$ in $H^{1}\left(X, \Theta_{X}\right)$. Note that if $X=\breve{H} \times \mathrm{U}(1)$, this summand is the entire $H^{1}\left(X, \Theta_{X}\right)$.

Let us review Kuranishi Theory briefly to set up notation. Let $\left\{\beta_{1}, \ldots, \beta_{N}\right\}$ be an orthonormal basis of the harmonic representatives of $H^{1}\left(X, \Theta_{X}\right)$. For any vector $\mathbf{t}=\left(t_{1}, \ldots, t_{N}\right)$ in $\mathbf{C}^{N}$, let $\phi_{1}(\mathbf{t})=t_{1} \beta_{1}+\ldots+t_{N} \beta_{N}$. Let $\mathcal{G}$ be the Green's operator and $\bar{\partial}^{*}$ be the adjoint operator of the $\bar{\partial}$-operator on $X$ with respect to the Hermitian metric $h$ previously defined. For $v \geq 2$, define inductively

$$
\phi_{\nu}(\mathbf{t})=\frac{1}{2} \sum_{\mu=1}^{\nu-1} \bar{\partial}^{*} \mathcal{G}\left\{\phi_{\mu}(\mathbf{t}), \phi_{\nu-\mu}(\mathbf{t})\right\},
$$


where the operator $\{$,$\} is the Nijenhuis bracket. Consider the formal sum \phi(t)=$ $\sum_{\nu \geq 1} \phi_{\nu}$. Let $\left\{\gamma_{1}, \ldots, \gamma_{M}\right\}$ be an orthonormal basis for the space of harmonic $(0,2)-$ forms with values in $\Theta_{X}$. Define $f_{k}(\mathbf{t})=\left(\{\phi(\mathbf{t}), \phi(\mathbf{t})\}, \gamma_{k}\right)$. According to the Kuranishi theory, there exists $\epsilon$ such that $\left\{\mathbf{t} \in \mathbf{C}^{N}:|\mathbf{t}|<\epsilon, f_{1}(\mathbf{t})=0, \ldots, f_{M}(\mathbf{t})=0\right\}$ forms a locally complete family of deformations of $X[\mathbf{1 5}$, Theorem 1].

Recall that $\left\{\bar{\omega}_{n+k}: 1 \leq k \leq m\right\}$ is a basis for $\Omega$. Let $v_{n+k}:=\bar{\omega}_{n+k} \otimes\left(H_{0}-i H_{1}\right)$. Then $\left\{v_{n+k}: 1 \leq k \leq m\right\}$ is a basis for $\Omega \otimes \mathfrak{v}_{0}$. Denote $\mu_{j k}=\frac{1}{2}\left(\bar{\omega}_{j} \otimes\left(X_{k}-i Y_{k}\right)+\bar{\omega}_{k} \otimes\right.$ $\left(X_{j}-i Y_{j}\right)$ ). Then $\left\{\mu_{j k}: 1 \leq j \leq k \leq n\right\}$ forms a basis for $\mathfrak{t}^{*(0,1)} \odot \mathfrak{t}^{1,0}$. As in Proposition 1, we use the Chern connection $\nabla$ with respect to the Hermitian metric $h$ to develop the harmonic theory on $X$. We have seen in the same proposition that

$$
\bar{\partial}^{\nabla}\left(X_{j}-i Y_{j}\right)=2 i\left(H_{0}-i H_{1}\right) \otimes \bar{\omega}_{j}, \quad \bar{\partial}^{\nabla}\left(H_{2 \ell}-i H_{2 \ell+1}\right)=0 .
$$

Since $\bar{\omega}_{\ell}$ for $1 \leq \ell \leq n+m$ are holomorphic,

$$
\bar{\partial}^{\nabla} v_{n+k}=0, \quad \bar{\partial}^{\nabla} \mu_{j k}=-i\left(\bar{\omega}_{j} \wedge \bar{\omega}_{k}+\bar{\omega}_{k} \wedge \bar{\omega}_{j}\right) \otimes\left(H_{0}-i H_{1}\right)=0 .
$$

With respect to the metric $h$, we need to compute $\bar{\partial}^{\nabla *} v_{n+k}$ and $\bar{\partial}^{\nabla *} \mu_{j k}$.

Lemma 6. The sections $v_{n+k}$ and $\mu_{j k}$ are harmonic.

Proof. Recall that a smooth frame of $(1,0)$-vectors is

$$
X_{j}-i Y_{j}, 1 \leq j \leq n, \quad H_{2 \ell}-i H_{2 \ell+1}, 0 \leq \ell \leq m-1, \quad \mathfrak{g}_{+}=\left\{W_{1}, \ldots, W_{N}\right\} .
$$

If $Y$ is a $(1,0)$-vector field, then there are smooth functions $a_{j}, c_{\ell}$ and $b_{\alpha}$ such that

$$
Y=\sum_{j} a_{j}\left(X_{j}-i Y_{j}\right)+\sum_{\ell} c_{\ell}\left(H_{2 \ell}-i H_{2 \ell+1}\right)+\sum_{\alpha} b_{\alpha} W_{\alpha} .
$$

Due to (45),

$$
\begin{aligned}
\bar{\partial}^{\nabla} Y= & \sum_{j} \bar{\partial} a_{j} \otimes\left(X_{j}-i Y_{j}\right)+\sum_{\ell} \bar{\partial} c_{\ell} \otimes\left(H_{2 \ell}-i H_{2 \ell+1}\right)+\sum_{\alpha} \bar{\partial} b_{\alpha} \otimes W_{\alpha} \\
& +\sum_{j} 2 i a_{j} \bar{\omega}_{j} \otimes\left(H_{0}-i H_{1}\right)+\sum_{\alpha} b_{\alpha} \bar{\partial}^{\nabla} W_{\alpha} .
\end{aligned}
$$

Since $H_{0}-i H_{1}$ is left-invariant, its length is constant. Therefore, with respect to the $L^{2}$-norm $\langle\cdot, \cdot\rangle_{L^{2}}$ on $\check{H} \times G$,

$$
\begin{aligned}
& \left\langle\bar{\partial}^{\nabla *} v_{n+k}, Y\right\rangle_{L^{2}}=\left\langle\bar{\omega}_{n+k} \otimes\left(H_{0}-i H_{1}\right), \bar{\partial}^{\nabla} Y\right\rangle_{L^{2}} \\
& \quad=\left\langle\bar{\omega}_{n+k}, \bar{\partial} c_{0}\right\rangle_{L^{2}}\left\|H_{0}-i H_{1}\right\|_{L^{2}}=\left\langle\bar{\partial}^{*} \bar{\omega}_{n+k}, c_{0}\right\rangle_{L^{2}}\left\|H_{0}-i H_{1}\right\|_{L^{2}} .
\end{aligned}
$$

Let $\left\{\omega_{k}, \omega_{n+k}, \omega_{\alpha}\right\}$ be the co-frame for $(1,0)$-vectors with respect to the basis (47) and $\left\{\bar{\omega}_{k}, \bar{\omega}_{n+k}, \bar{\omega}_{\alpha}\right\}$ be the co-frame for $(0,1)$-vectors. Recall that [18, pp. 166-168] $\bar{\partial}^{*}=-\bar{*} \circ \bar{\partial} \circ \bar{*}$

Let $\Xi$ be the pull-back of the volume form on the Borel flag $G / U$ and $\Pi$ the pull-back of the volume form on the torus $T^{2 n}$. Then

$$
\bar{\partial}^{*} \bar{\omega}_{n+k}=-\bar{*} \bar{\partial} * \omega_{n+k}= \pm \bar{\partial} \bar{\partial}\left(\bar{\omega}_{n+k} \wedge_{j \neq k} \omega_{n+j} \wedge \bar{\omega}_{n+j} \wedge \Pi \wedge \Xi\right) .
$$


It is apparent that $\Pi$ and $\Xi$ are closed. As $d \omega_{n+\ell}$ is a curvature form, $d \omega_{n+\ell} \wedge \Pi \wedge \Xi=$ 0 . Therefore, $\bar{\partial}^{*} \bar{\omega}_{n+k}=0$, and hence $\bar{\partial}^{\nabla *} v_{n+k}=0$.

Similarly,

$$
\bar{\partial}^{*} \bar{\omega}_{k}=-\bar{*} \bar{\partial} * \omega_{k}= \pm \bar{*} \bar{\partial}\left(\bar{\omega}_{k} \wedge_{j \neq k} \omega_{j} \wedge \bar{\omega}_{j} \wedge_{\ell} \omega_{n+\ell} \wedge \bar{\omega}_{n+\ell} \wedge \Xi\right) .
$$

Note that $\omega_{j}$ and $\bar{\omega}_{j}$ are closed forms. Also, $\omega_{n+\ell}$ are connection forms over $T^{2 n} \times G$ and $\bar{\omega}_{n+\ell}$ are holomorphic. Therefore, $\bar{\partial}^{*} \bar{\omega}_{k}=0$. Hence

$$
\begin{aligned}
\left\langle\bar{\partial}^{\nabla *} v_{n+k}, Y\right\rangle_{L^{2}}=\left\langle v_{n+k}, \bar{\partial}^{\nabla} Y\right\rangle_{L^{2}} \\
\quad=\left\langle\bar{\omega}_{j} \otimes\left(X_{k}-i Y_{k}\right)+\bar{\omega}_{k} \otimes\left(X_{j}-i Y_{j}\right), \bar{\partial}^{\nabla} Y\right\rangle_{L^{2}} \\
\quad=\left\langle\bar{\omega}_{j} \otimes\left(X_{k}-i Y_{k}\right)+\bar{\omega}_{k} \otimes\left(X_{j}-i Y_{j}\right), \bar{\partial} a_{\ell} \otimes\left(X_{\ell}-i Y_{\ell}\right)\right\rangle_{L^{2}} \\
\quad=\left\langle\bar{\omega}_{j}, \bar{\partial} a_{k}\right\rangle+\left\langle\bar{\omega}_{k}, \bar{\partial} a_{j}\right\rangle=a_{k} \bar{\partial}^{*} \bar{\omega}_{j}+a_{j} \bar{\partial}^{*} \bar{\omega}_{k}=0 .
\end{aligned}
$$

and so the proof is complete.

Now, we are ready to prove the following result.

Proposition 4. Every element in $\Omega \otimes \mathfrak{v}_{0} \oplus \mathfrak{t}^{*(0,1)} \odot \mathfrak{t}^{1,0}$ in $H^{1}\left(X, \Theta_{X}\right)$ is tangent to a 1-parameter family of deformations.

Proof. Every element $\phi_{1}=\sum_{\ell=1}^{m} t_{\ell} v_{n+\ell}+\sum_{1 \leq j \leq k}^{n} t_{j k} \mu_{j k}$ in $\Omega \otimes \mathfrak{v}_{0} \oplus \mathfrak{t}^{*(0,1)} \odot \mathfrak{t}^{1,0}$ is harmonic (Lemma 6). By Lemma 3 the Nijenhuis bracket $\left\{\phi_{1}, \phi_{1}\right\}$ vanishes. The proof is complete.

We are now able to sum up the main results in the following theorems.

THEOREM 1. Let $G$ be a compact semi-simple Lie group. Let $X=\breve{H} \times G$ be the compact quotient of the complex manifold $H_{2 n+1} \times G$ with a left-invariant complex structure determined by a maximal torus $U$ in $G$. Then every element in $\mathcal{N}$ and every simple element $\bar{\omega} \otimes V$ in $\mathcal{S}$ is tangent to a 1-parameter family of deformations. Moreover,

$$
\mathcal{P}=\Omega \otimes \mathfrak{v}_{0} \oplus \mathfrak{t}^{*(0,1)} \odot \mathfrak{t}^{1,0} \oplus\left(\Omega \oplus \mathfrak{t}^{*(0,1)}\right) \otimes\left(\mathfrak{v}_{1} \oplus \mathfrak{u}_{\mathbf{C}}\right)
$$

parameterizes a local deformation space, which is maximal in $\mathcal{N} \oplus \mathcal{S}$, if $H^{1}\left(X, \mathcal{O}_{X}\right)$ is at least of dimension two.

Proof. By Proposition 3 and Proposition 4, every element of the form $\bar{\omega} \otimes V$ in $\mathcal{S}$ and every element in $\mathcal{N}$ is tangent to a 1-parameter family of deformations. By Lemma $4,\{\mathcal{N}, \mathcal{S}\}=0$. It follows that every element in $\mathcal{P}$ is a local deformation space, and so the proof is complete.

Note that elements in $H^{0}\left(X, \Theta_{X}\right)$ and in the space $\mathcal{P}$ are all invariant of $C \times U$. By Cathelineau's equivariant deformation theory [6], we obtain the following version of the last theorem.

THEOREM 2. The space of $C \times U$-equivariant deformations of $X=\check{H} \times G$ is locally parametrized by $\mathcal{P}=\Omega \otimes \mathfrak{v}_{0} \oplus \mathfrak{t}^{*(0,1)} \odot \mathfrak{t}^{1,0} \oplus\left(\Omega \oplus \mathfrak{t}^{*(0,1)}\right) \otimes\left(\mathfrak{v}_{1} \oplus \mathfrak{u}_{\mathbf{C}}\right)$, which is maximal in the local parameter space of all deformations. 
4. Moduli of Kodaira manifolds. For Kodaira manifolds $G=\mathrm{U}(1)$. Therefore the component $\mathcal{S}$ of $H^{1}\left(X, \Theta_{X}\right)$ is equal to zero. We have the following result.

THEOREM 3. Let $X=\breve{H} \times \mathrm{U}(1)$ be the compact quotient of the complex manifold $H_{2 n+1} \times \mathrm{U}(1)$ with a left-invariant complex structure. Then every element in $H^{1}\left(X, \Theta_{X}\right)$ is tangent to a 1-parameter family of deformations. Moreover,

$$
\Omega \otimes \mathfrak{v}_{0} \oplus \mathfrak{t}^{*(0,1)} \odot \mathfrak{t}^{1,0}
$$

parametrizes a local maximal deformation space.

Based on this infinitesimal result, we extend our scope to a global one with some limitation.

4.1. Invariant complex structures. To realize the deformations, we recall that elements in $H^{1}(X, \Theta)$ are $\Theta$-valued $(0,1)$-forms

$$
\theta=s_{j k} \bar{\omega}_{j} \otimes\left(X_{k}-i Y_{k}\right)+s(\gamma-i d t) \otimes(Z-i T)
$$

where $s_{j k}=s_{k j}$. The forms $\theta$ act as deformations by changing the distribution of $(0,1)$ vectors. For any $t \in \mathbf{R}$, define

$$
\begin{aligned}
V_{l}(\theta, t) & =X_{l}+i Y_{l}+t \theta\left(X_{l}+i Y_{l}\right)=X_{l}+i Y_{l}+t \sum_{k} s_{l k}\left(X_{k}-i Y_{k}\right), \\
V_{n+1}(\theta, t) & =Z+i T+t \theta(Z+i T)=Z+i T+t s(Z-i T) .
\end{aligned}
$$

For the distribution defined by $D=\left\{V_{1}(\theta, t), \ldots, V_{n}(\theta, t)\right\}$ to define a complex structure $J(\theta, t)$ it is necessary that $D \oplus \bar{D}=\left(\mathfrak{h}_{2 n+1} \oplus \mathfrak{t}_{1}\right)_{\mathbf{C}}$ (non-degeneracy condition.) All complex structures constructed in this way descend to the quotient space $X$. All of these deformations are left-invariant in the sense that this is the quotient of a left-invariant complex structure on the universal covering.

We now extend the complex parameters $\left(s_{j k}, s\right)$ as follows. Define a distribution spanned by

$$
\begin{aligned}
V_{l} & =\sum_{k} a_{l k}\left(X_{k}+i Y_{k}\right)+\sum_{k} b_{l k}\left(X_{k}-i Y_{k}\right), \\
V_{n+1} & =a(Z+i T)+b(Z-i T)
\end{aligned}
$$

Let $A$ and $B$ be the matrices whose entries are $a_{j k}$ and $b_{j k}$ respectively. The matrix $(A \mid B)$, together with $a$ and $b$, determines the above distribution. Since a complex structure is defined by the span of the vectors, we treat $(A \mid B)$ as an element in the Grassmannian of n-planes in $\mathbf{C}^{2 n}$. The parameter $[a, b]$ is considered as an element of $\mathbf{C P}^{1}$. Therefore the parameter space of all invariant complex structure is contained in $\operatorname{Gr}\left(n, \mathbf{C}^{2 n}\right) \times \mathbf{C} \mathbf{P}^{1}$. For the distribution $D$ to be integrable it must be closed under the bracket. This is equivalent to

$$
\sum_{k} b_{j k} a_{l k}=\sum_{k} a_{j k} b_{l k}, \quad \text { or equivalently } \quad B A^{T}=A B^{T} .
$$


For the distribution to be nondegenerate, and to induce the same orientation as $X_{1} \wedge Y_{1} \wedge \ldots \wedge X_{n} \wedge Y_{n} \wedge Z \wedge T$ we must have

$$
\left(|a|^{2}-|b|^{2}\right) \operatorname{det}\left(\frac{A}{B} \frac{B}{A}\right)>0 .
$$

This condition is equivalent to $\left(|a|^{2}-|b|^{2}\right) \operatorname{det}\left(A A^{*}-B B^{*}\right)>0$. The space of parameters has two components

$$
\begin{aligned}
& \check{\mathcal{U}}=\left\{(A \mid B) \times[a, b]:|a|^{2}-|b|^{2}>0, \operatorname{det}\left(A A^{*}-B B^{*}\right)>0, B A^{T}=A B^{T}\right\} . \\
& \check{\mathcal{V}}=\left\{(A \mid B) \times[a, b]:|a|^{2}-|b|^{2}<0, \operatorname{det}\left(A A^{*}-B B^{*}\right)<0, B A^{T}=A B^{T}\right\} .
\end{aligned}
$$

4.2. Identification of equivalent structures. Given two different parameters $\left(A^{\prime} \mid B^{\prime}\right) \times\left[a^{\prime}, b^{\prime}\right] \neq(A \mid B) \times[a, b]$, the corresponding complex structures on $X$ are equivalent if and only if there is a diffeomorphism $F$ of $X$ so that the distribution $(A \mid B) \times[a, b]$ is sent to $\left(A^{\prime} \mid B^{\prime}\right) \times\left[a^{\prime}, b^{\prime}\right]$. Define the diffeomorphism

$$
f\left(x_{j}, y_{j}, z, t\right)=F(0,0,0,0)^{-1} * F\left(x_{j}, y_{j}, z, t\right) .
$$

The diffeomorphism $f$ sends $(A \mid B) \times[a, b]$ to $\left(A^{\prime}, B^{\prime}\right) \times\left[a^{\prime}, b^{\prime}\right]$, and is a group isomorphism of the lattice $\check{\Gamma}$. Let $G_{k}=f\left(g_{k}\right)$. Then there exist integers $\eta_{k, l}$ such that

$$
G_{k}=\left(\prod_{j=1}^{n} g_{2 j-1}^{\eta_{2 j-1, k}} g_{2 j}^{\eta_{2 j, k}}\right) g_{2 n+1}^{\eta_{2 n+1, k}} g_{2 n+2}^{\eta_{2 n+2, k}} .
$$

Since $G_{2 n+1}$ and $G_{2 n+2}$ are in the center,

$$
G_{2 n+1}=g_{2 n+1}^{\eta_{2 n+1,2 n+1}} g_{2 n+2}^{\eta_{2 n+2,2 n+1}}, \quad G_{2 n+2}=g_{2 n+1}^{\eta_{2 n+1,2 n+2}} g_{2 n+2}^{\eta_{2 n+2,2 n+2}} .
$$

The following proposition describes the commuting properties

Proposition 5. For any integers $h$ and $k, \quad G_{2 j}^{k} G_{2 j-1}^{h}=G_{2 n+1}^{-h k m} G_{2 j-1}^{h} G_{2 j}^{k}$.

Now, using

$$
G_{2 n+1}^{m}=G_{2 j-1} G_{2 j} G_{2 j-1}^{-1} G_{2 j}^{-1}
$$

we can compute

$$
\begin{aligned}
& g_{2 n+1}^{m\left(\eta_{2 n+1,2 n+1}\right)} g_{2 n+2}^{m\left(\eta_{2 n+2,2 n+1}\right)}=G_{2 n+1}^{m}=G_{2 l-1} G_{2 l} G_{2 l-1}^{-1} G_{2 l}^{-1} \\
& \quad=\prod_{j=1}^{n}\left(g_{2 j-1}^{\eta_{2 j-1,2 l-1}} g_{2 j}^{\eta_{2 j, 2 l-1}} g_{2 j-1}^{\eta_{2 j-1,2 l}} g_{2 j}^{\eta_{2 j, 2 l}} g_{2 j}^{-\eta_{2 j, 2 l-1}} g_{2 j-1}^{-\eta_{2 j-1,2 l-1}} g_{2 j}^{-\eta_{2 j, 2 l}} g_{2 j-1}^{-\eta_{2 j-1,2 l}}\right) \\
& \quad=g_{2 n+1}^{m \sum_{j=1}^{n}\left(\eta_{2 j-1,2 l-1} \eta_{2 j, 2 l}-\eta_{2 j, 2 l-1} \eta_{2 j-1,2 l}\right)} .
\end{aligned}
$$

It follows that $\eta_{2 n+2,2 n+1}=0$ and that for $1 \leq l \leq n$

$$
\sum_{j=1}^{n}\left(\eta_{2 j-1,2 l-1} \eta_{2 j, 2 l}-\eta_{2 j, 2 l-1} \eta_{2 j-1,2 l}\right)=\eta_{2 n+1,2 n+1} .
$$


For all other pairs $1 \leq k, l \leq 2 n$, the identity $G_{k} G_{l} G_{k}^{-1} G_{l}^{-1}=i d$ leads to

$$
\sum_{j=1}^{n}\left(\eta_{2 j-1, l} \eta_{2 j, k}-\eta_{2 j, l} \eta_{2 j-1, k}\right)=0
$$

Let $S$ be the matrix $\left(\eta_{j, k}\right)_{1 \leq j, k \leq 2 n}$, and let $D$ be the $(2 n \times 2 n)$-matrix with $2 \times 2$-block $\left(\begin{array}{cc}0 & 1 \\ -1 & 0\end{array}\right)$ along the diagonal, the last two identities together are equivalent to

$$
S^{T} D S=\eta_{2 n+1,2 n+1} D
$$

Since restriction of $f$ to the center of $\check{\Gamma}$ is an isomorphism, the matrix

$$
\left(\begin{array}{cc}
\eta_{2 n+1,2 n+1} & \eta_{2 n+1,2 n+2} \\
0 & \eta_{2 n+2,2 n+1}
\end{array}\right)
$$

is in $G L(2, \mathbf{Z})$. Therefore $\left|\eta_{2 n+1,2 n+1}\right|=\left|\eta_{2 n+2,2 n+2}\right|= \pm 1$.

Now since $\log G_{k}=d f\left(\log g_{k}\right)$, we can calculate the matrix of $d f$.

$$
\begin{aligned}
\log G_{l}= & \log \left[\left(\prod_{j=1}^{n} g_{2 j-1}^{\eta_{2 j-1, l}} g_{2 j}^{\eta_{2 j, l}}\right) g_{2 n+1}^{\eta_{2 n+1, l}} g_{2 n+2}^{\eta_{2 n+2, l}}\right] \\
= & \sum_{j=1}^{n} \log \left(g_{2 j-1}^{\eta_{2 j-1, l}} g_{2 j}^{\eta_{2 j, l}}\right)+\log g_{2 n+1}^{\eta_{2 n+1, l}}+\log g_{2 n+2}^{\eta_{2 n+2, l}} \\
= & \sum_{j=1}^{n} \log \left(g_{2 j-1}^{\eta_{2 j-1, l}}\right)+\sum_{j=1}^{n} \log \left(g_{2 j}^{\eta_{2 j, l}}\right) \\
& +\sum_{j=1}^{n}\left[\log \left(g_{2 j-1}^{\eta_{2 j-1, l}}\right), \log \left(g_{2 j}^{\eta_{2 j, l}}\right)\right]+\log g_{2 n+1}^{\eta_{2 n+1, l}}+\log g_{2 n+2}^{\eta_{2 n+2, l}} \\
= & \sum_{j=1}^{n} \eta_{2 j-1, l} X_{j}+\sum_{j=1}^{n} \eta_{2 j, l} Y_{j}+\left(\eta_{2 n+1, l}+\sum_{j=1}^{n} \eta_{2 j-1, l} \eta_{2 j, l}\right) Z+\eta_{2 n+2, l} T .
\end{aligned}
$$

We then obtain $d f\left(X_{k}\right)=\log \left(G_{2 k-1}\right)$ and $d f\left(Y_{k}\right)=\log \left(G_{2 k}\right)$. Finally,

$$
\begin{aligned}
& d f(Z)=\log G_{2 n+1}=\log g_{2 n+1}^{\eta_{2 n+1,2 n+1}}=\eta_{2 n+1,2 n+1} Z, \\
& d f(T)=\log G_{2 n+2}=\log g_{2 n+1}^{\eta_{2 n+1,2 n+2}}+\log g_{2 n+2}^{\eta_{2 n+2,2 n+2}}=\eta_{2 n+1,2 n+2} Z+\eta_{2 n+2,2 n+2} T .
\end{aligned}
$$

To summarize, let $e=\eta_{2 n+1,2 n+1}, \alpha=\eta_{2 n+1,2 n+2}$, and $\varepsilon=\eta_{2 n+2,2 n+2}$. Define the vectors

$$
\eta_{1}=\left[\eta_{2 n+1, k}+\sum_{j=1}^{n} \eta_{2 j-1, k} \eta_{2 j, k}\right]_{1 \leq k \leq 2 n}, \quad \eta_{2}=\left[\eta_{2 n+2, k}\right]_{1 \leq k \leq 2 n} .
$$


The matrix of $d f$ with respect to the ordered basis $<X_{k}, Y_{k}, Z, T>$ is

$$
\left(\begin{array}{ccc}
S & 0 & 0 \\
& 0 & 0 \\
\eta_{1}^{T} & e & \alpha \\
\eta_{2}^{T} & 0 & \varepsilon
\end{array}\right) .
$$

Suppose the diffeomorphism $f$ sends the distribution spanned by $\left(V_{1}, \ldots\right.$, $\left.V_{n}, V_{n+1}\right)$ determined by $(A \mid B) \times[a, b]$ to another distribution $\left(W_{1}, \ldots, W_{n}, W_{n+1}\right)$ determined by $\left(A^{\prime} \mid B^{\prime}\right) \times\left[a^{\prime}, b^{\prime}\right]$. Since the center is preserved, set $V_{n+1}^{\prime}=d f\left(V_{n+1}\right)$, and we may assume that $W_{n+1}=V_{n+1}^{\prime}$. Then define $V_{k}^{\prime}$ by $d f\left(V_{k}\right)=V_{k}^{\prime}+r_{k} V_{n+1}^{\prime}$. It can be checked that $\left(V_{1}^{\prime}, \ldots, V_{n}^{\prime}\right)$ is a linearly independent set. Therefore, the span of $\left(V_{1}^{\prime}, \ldots, V_{n}^{\prime}\right)$ is identical to the span of $\left(W_{1}, \ldots, W_{n}\right)$.

Define $\beta_{2 j-1, l}=a_{l, j}+b_{l, j}$ and $\beta_{2 j, l}=i\left(a_{l j}-b_{l j}\right)$. Then

$$
V_{l}=\sum_{j=1}^{n} \beta_{2 j-1, l} X_{j}+\sum_{j=1}^{n} \beta_{2 j, l} Y_{j} .
$$

For $1 \leq l \leq n$, let $\beta_{l}$ be the vector in $\mathbf{C}^{2 n}$ whose $\mathrm{k}$-th coordinate is $\beta_{k, l}$. Similarly define $\beta_{k, l}^{\prime}$ so that $V_{l}^{\prime}=\sum_{j=1}^{n} \beta_{2 j-1, l}^{\prime} X_{j}+\sum_{j=1}^{n} \beta_{2 j}^{\prime} Y_{j}+r_{l} V_{n+1}^{\prime}$. Then

$$
\begin{aligned}
\left(\begin{array}{ccc}
S & 0 & 0 \\
& 0 & 0 \\
\eta_{1}^{T} & e & \alpha \\
\eta_{2}^{T} & 0 & \varepsilon
\end{array}\right)\left(\begin{array}{cccc}
\beta_{1} & \cdots & \beta_{n} & 0 \\
0 & \cdots & 0 & a+b \\
0 & \cdots & 0 & i(a-b)
\end{array}\right) \\
=\left(\begin{array}{cccc}
\beta_{1}^{\prime} & \cdots & \beta_{n}^{\prime} & 0 \\
r_{1}\left(a^{\prime}+b^{\prime}\right) & \cdots & r_{n}\left(a^{\prime}+b^{\prime}\right) & a^{\prime}+b^{\prime} \\
i r_{1}\left(a^{\prime}-b^{\prime}\right) & \cdots & i r_{n}\left(a^{\prime}-b^{\prime}\right) & i\left(a^{\prime}-b^{\prime}\right)
\end{array}\right) .
\end{aligned}
$$

Proposition 6. The distribution $(A \mid B) \times[a, b]$ is equivalent to $\left(A^{\prime} \mid B^{\prime}\right) \times\left[a^{\prime}, b^{\prime}\right]$ if and only if there is a matrix $S$ and integers $e, \alpha$, and $\varepsilon$ such that:

(i) $S$ satisfies (65), equivalently $S^{T} D S=e D$.

(ii) $S \beta_{l}=\beta_{l}^{\prime}$ for $1 \leq l \leq n$.

(iii) $e(a+b)+i \alpha(a-b)=a^{\prime}+b^{\prime}$ and $\varepsilon(a-b)=a^{\prime}-b^{\prime}$.

(iv) $|e|=|\epsilon|= \pm 1$.

Proof. If $\left(A^{\prime} \mid B^{\prime}\right) \times\left[a^{\prime}, b^{\prime}\right]$ is equivalent to $(A \mid B) \times[a, b]$ then the equations are clearly satisfied because of the calculations above. Conversely, given such an $S, e, \alpha$, and $\varepsilon$ we can choose all $r_{i}=0$ because the span of $\left(V_{1}+r_{1} V_{n+1}, \ldots, V_{n}+r_{n} V_{n+1}, V_{n+1}\right)$ is the same as that of $\left(V_{1}, \ldots, V_{n}, V_{n+1}\right)$, and we can freely choose the remaining parameters so that $\eta_{1}^{T}=\eta_{2}^{T}=0$, thus producing a diffeomorphism that sends $(A \mid B) \times$ $[a, b]$ to $\left(A^{\prime} \mid B^{\prime}\right) \times\left[a^{\prime}, b^{\prime}\right]$. This completes the proof.

The first consequence of the above proposition is a distinction between two scenarios in analyzing the moduli space.

COROLlary 1. When $n$ is odd, every complex structure in the component $\hat{\mathcal{V}}$ of the parameter space is equivalent to a complex structure in $\hat{\mathcal{U}}$. When $n$ is even, complex structures in $\hat{\mathcal{V}}$ are not equivalent to any complex structures in $\hat{\mathcal{U}}$. 
Proof. Suppose that $\left(A^{\prime} \mid B^{\prime}\right) \times\left[a^{\prime}, b^{\prime}\right]$ is a parameter in $\hat{\mathcal{V}}$ and $(A \mid B) \times[a, b]$ is in $\hat{\mathcal{U}}$. If there is an identification of these two parameter points, then $\operatorname{det} S=-1$. On the other hand, $D$ is a symplectic form and $S$ is a symplectic map up to the factor $e$ as seen in (i) of the last proposition. Therefore, $\operatorname{det} S=e^{n}$. When $n$ is even, $\operatorname{det} S=1$. Therefore, this is impossible to identify parameters in $\hat{\mathcal{V}}$ with those in $\hat{\mathcal{U}}$.

When $n$ is odd, we consider a diffeomorphism $f$ whose differential has the 2-by-2 block

$$
\left(\begin{array}{cc}
-1 & 0 \\
0 & 1
\end{array}\right)
$$

along its diagonal and zero elsewhere. It identifies $\hat{\mathcal{V}}$ with $\hat{\mathcal{U}}$. This completes the proof.

4.3. Describing the moduli space when $n$ is odd. We conclude from the last corollary that the moduli space in this case is a quotient of the product of $\mathcal{B} \times \mathcal{U}$ where

$$
\mathcal{B}=\left\{[a, b] \in \mathbf{C P}^{1}:|a|^{2}-|b|^{2}>0\right\}
$$

and

$$
\mathcal{U}=\left\{(A \mid B) \in \operatorname{Gr}\left(n, \mathbf{C}^{2 n}\right): \operatorname{det}\left(A A^{*}-B B^{*}\right)>0, B A=A B^{T}\right\} .
$$

Consider the group $\operatorname{Sp}(2 n, \mathbf{Z})=\left\{S \in \mathrm{GL}(2 n, \mathbf{Z}): S^{T} D S=D\right\}$. In view of the last proposition, $\operatorname{Sp}(2 n, \mathbf{Z})$ can be regarded as subgroup of automorphisms of $\check{\Gamma}$ through the map

$$
S \mapsto\left(\begin{array}{cc}
S & 0 \\
0 & \mathrm{I}
\end{array}\right)
$$

Using the inhomogeneous coordinate $b / a, \mathcal{B}$ can be identified with the unit disc $\{b / a \in \mathbf{C}:|b / a|<1\}$. The map $(a, b) \mapsto \frac{a+b}{a-b}$ sends the open unit disk to the right half plane. In addition, if a diffeomorphism with the properties $S=\mathrm{I}, \eta_{1}=\eta_{2}=0$, and $e=\varepsilon$ identifies the complex structures $(A \mid B) \times[a, b]$ and $(A \mid B) \times\left[a^{\prime}, b^{\prime}\right]$ then it satisfies (iii) of the last proposition. It follows that

$$
\frac{a^{\prime}+b^{\prime}}{a^{\prime}-b^{\prime}}=\left(\frac{a+b}{a-b}\right)+i \frac{\alpha}{\varepsilon} .
$$

Therefore, The fundamental domain of the translation in the imaginary direction is the strip

$$
\{r+i \theta \in \mathbf{C}: r>0,0 \leq \theta<1\} .
$$

Taking $e^{-2 \pi(r+i \theta)}$, we identify the fundamental domain to the punctured disc. Therefore, the moduli space is the product: $\{z \in \mathbf{C}: 0<|z|<1\} \times \mathcal{U} / \operatorname{Sp}(2 n, \mathbf{Z})$.

To describe the quotient space $\mathcal{U} / \operatorname{Sp}(2 n, \mathbf{Z})$, we consider the transformation

$$
\phi:(A \mid B) \rightarrow(A+B \mid i(A-B))
$$


This map preserves the condition $A B^{T}=B A^{T}$ and transforms the determinants as follows.

$$
\operatorname{det}\left(A A^{*}-B B^{*}\right)=\operatorname{det}\left(2 i\left((A+B)(i(A-B))^{*}-i(A-B)(A+B)^{*}\right)\right) .
$$

Define

$$
\mathcal{H}_{n}=\left\{(A \mid B) \in \operatorname{Gr}\left(n, \mathbf{C}^{2 n}\right) \mid A B^{T}=B A^{T}, \operatorname{det}\left[i\left(A B^{*}-B A^{*}\right)\right] \neq 0\right\}
$$

According to [3, Proposition 1.2, p. 181], $\mathcal{H}_{n}$ is a homogeneous space with respect to the group $\operatorname{Sp}(2 n, \mathbf{C})$ of dimension $n(n+1) / 2$. Define

$$
\mathcal{H}_{n, k}=\left\{(A \mid B) \in \mathcal{H}_{n}: \operatorname{ind}\left[i\left(A B^{*}-B A^{*}\right)\right]=k\right\} .
$$

By [3, Proposition 1.5, p. 183], this space is the flag domain $\operatorname{Sp}(2 n, \mathbf{R}) / \mathrm{U}_{n-k, k}(\mathbf{C})$.

We identify $\mathcal{U}$ with $\phi(\mathcal{U})$. Let $n=2 \ell+1$. Since $\operatorname{det}\left[i\left(A B^{*}-B A^{*}\right)\right]>0$, the number of negative eigenvalues of $\left[i\left(A B^{*}-B A^{*}\right)\right]$ is even. Hence, its index is necessarily odd. Therefore, $\mathcal{U}$ is the disjoint union:

$$
\mathcal{U}=\cup_{j=0}^{\ell} \mathcal{H}_{2 \ell+1,2 \ell+1-4 j} .
$$

Finally, by [3, Theorems 4.2 and 4.3, p. 219], the action of $\operatorname{Sp}(2 n, \mathbf{Z})$ gives us a description of the topological coarse moduli space of complex structures on $\Gamma \backslash H_{2 n+1} \times$ $S^{1}$ when $n$ is odd.

THEOREM 4. The moduli space of invariant complex structures on the complex $2(\ell+1)$-dimensional Kodaira manifold $\Gamma \backslash H_{4 \ell+3} \times S^{1}$ is the topological space

$$
\mathcal{M}=\cup_{j=0}^{\ell}\left(\mathcal{H}_{2 \ell+1,2 \ell+1-4 j} / \mathrm{Sp}(4 \ell+2, \mathbf{Z})\right) \times D^{*}
$$

where $D^{*}$ is the punctured unit disc.

Note that except for the Siegel spaces $\mathcal{H}_{n, 0} / \operatorname{Sp}(2 n, \mathbf{Z})$ and $\mathcal{H}_{n, n} / \operatorname{Sp}(2 n, \mathbf{Z})$, the spaces $\mathcal{H}_{n, k} / \operatorname{Sp}(2 n, \mathbf{Z})$ are non-Hausdorff. In particular we don't have a coarse moduli space of complex structures when $\ell \geq 1$.

REMARK 1. The last theorem demonstrates that when $\ell=0$ the moduli space is smooth. This is consistent with Borcea's work [4]. Given a complex structure described by the parameters $\left(\lambda, b_{11} ; \mu, b\right)$ or equivalently by the parameters $\left.\lambda^{\prime}, b_{11}^{\prime} ; \mu^{\prime}, b^{\prime}\right)$. Then

$$
\frac{\lambda^{\prime}+b_{11}^{\prime}}{\lambda^{\prime}-b_{11}^{\prime}}=\frac{\eta_{11}\left(\lambda+b_{11}\right)+i \eta_{12}\left(\lambda-b_{11}\right)}{-i \eta_{21}\left(\lambda+b_{11}\right)+\eta_{22}\left(\lambda-b_{11}\right)} .
$$

This is a modular transformation as $\eta_{11} \eta_{22}-\eta_{12} \eta_{21}=1$. If we consider the coordinate change

$$
\left(\lambda, b_{11} ; \mu, b\right) \mapsto\left(\lambda+b_{11}, \lambda-b_{11} ; \mu+b, \mu-b\right) \mapsto\left(\frac{\lambda+b_{11}}{\lambda-b_{11}}, \frac{\mu+b}{\mu-b}\right)
$$

the product of disks is mapped to the product of right half spaces. With respect to the quotient of the modular group, the quotient of the first factor becomes a complex plane. As in the general situation the second factor becomes the punctured disk. This is precisely the picture given by Borcea [4]. 
4.4. Describing the moduli space when $n$ is even. Set $n=2 \ell$. In this case, we need to consider both $\hat{\mathcal{V}}$ and $\hat{\mathcal{U}}$. As seen in the last section, the component $\hat{\mathcal{U}}$ is the product space $\mathcal{B} \times \mathcal{U}$ where $\mathcal{B}$ is again mapped to the punctured disk through equivalence. Consider the space

$$
\mathcal{H}_{2 \ell}=\left\{(A \mid B) \in \operatorname{Gr}\left(2 \ell, \mathbf{C}^{4 \ell}\right) \mid A B^{T}=B A^{T}, \operatorname{det}\left[i\left(A B^{*}-B A^{*}\right)\right] \neq 0\right\} .
$$

For elements in $\mathcal{U}, \operatorname{det}\left[i\left(A B^{*}-B A^{*}\right)\right]>0$. Therefore, the number of their negative eigenvalues is even. Given the dimension, it implies that the index is also even. Therefore

$$
\mathcal{U}=\cup_{j=0}^{\ell} \mathcal{H}_{2 \ell, 2 \ell-4 j}
$$
and

The component $\hat{\mathcal{V}}$ is the product of $\mathcal{C} \times \mathcal{V}$ where $\mathcal{C}=\left\{[a, b] \in \mathbf{C P}^{1}:|a|^{2}-|b|^{2}<0\right\}$

$$
\mathcal{V}=\left\{(A \mid B) \in G r\left(n, \mathbf{C}^{2 n}\right): \operatorname{det}\left(A A^{*}-B B^{*}\right)<0, B A=A B^{T}\right\}
$$

The space $\mathcal{C}$ is again mapped to the punctured disk via equivalence of complex structures. Via the map $\phi, \mathcal{V}$ is identified to

$$
\left\{(A \mid B) \in \mathcal{H}_{2 \ell} \mid \operatorname{det}\left[i\left(A B^{*}-B A^{*}\right)\right]<0\right\} .
$$

The determinant is negative if and only if there are odd number of negative eigenvalues. As the dimension of the matrices in questions is $2 \ell \times 2 \ell$, the index is even. Therefore,

$$
\mathcal{V}=\cup_{j=0}^{\ell-1} \mathcal{H}_{2 \ell, 2 \ell-2-4 j}
$$

It follows that $\mathcal{U} \cup \mathcal{V}=\cup_{j=0}^{2 \ell} \mathcal{H}_{2 \ell, 2 \ell-2 j}$. A general description of the moduli space in this case now is similar to that leading the previous theorem.

THEOREM 5. The moduli space of invariant complex structures on the complex $2 \ell+1$-dimensional Kodaira manifold $\Gamma \backslash H_{4 \ell+1} \times S^{1}$ is the topological space

$$
\mathcal{M}=\left(\cup_{j=0}^{2 \ell} \mathcal{H}_{2 \ell, 2 \ell-2 j} / \operatorname{Sp}(4 \ell, \mathbf{Z})\right) \times D^{*},
$$

where $D^{*}$ is the punctured unit disc.

REMARK 2. We learn that all small deformations of invariant complex structures on Kodaira manifolds are invariant. We then extend our moduli consideration to all invariant complex structures. However, we do not know if all deformation of invariant complex structures are invariant. Therefore, strictly speaking, we have not yet identified the full moduli for Kodaira manifolds. In the complex 2-dimensional case, this concern is removed due to the classification that all Kodaira manifolds are cocompact quotients [13].

REMARK 3. Our work leading to Theorem 1 is applicable when the Heisenberg group is replaced by a one-dimensional compact Abelian group U(1). For example, when the simple group $G$ is $\mathrm{SU}(\ell)$, we obtain the parameter space of deformations of the left-invariant complex structures on the Hopf manifolds: $\mathrm{U}(1) \times \mathrm{SU}(2 \ell)$. The case when $\ell=1$ was studied by several authors including Dabrowski [7].

Our computation also yields a parameter space for the deformations of the Kodaira-Hopf Manifold: $\Gamma \backslash H_{3} \times \mathrm{SU}(2)$. The manifold $Y=\Gamma \backslash H_{3} \times \mathrm{SU}(2)$ is an elliptic fibration over the product of the elliptic curve and the Riemann sphere. Taking 
further projection onto the elliptic curve, we obtain a fibration of Hopf surface with uniform complex structure. Taking the projection onto the Riemann sphere, we obtain a fibration of Kodaira surface with uniform complex structure.

By Theorem 1, a maximal parameter space is 6-dimensional. Among the six parameters of deformations, those in the nilpotent part are attributed to deformation of the Kodaira surfaces without changing the bundle structure over the Riemann sphere.

Acknowledgment. We are grateful to Simon Salamon for valuable discussions and to Galliano Valent for advice.

\section{REFERENCES}

1. E. Abbena, S. Garbiero and S. Salamon, Almost Hermitian geometry on six dimensional nilmanifolds, Ann. Scuola Norm. Sup. Pisa Cl. Sci. (4) 30 (2001), 147-170. 1984).

2. W. Barth, C. Peters and A. Van de Ven, Compact complex surfaces (Springer-Verlag, 1999).

3. C. Birkenhake and H. Lange, Complex tori, Progress in Mathematics 177 (Birkhäuser,

4. C. Borcea, Moduli for Kodaira surfaces, Compositio Math. 52 (1984), 373-380.

5. R. Bott, Homogeneous vector bundles, Ann. of Math. 66 (1958), 203-248.

6. J. L. Cathelineau, Deformations équivariantes d'espaces analytiques complexes compacts, Ann. Sci. Ecole Norm. Sup. (4) 11 (1978), 391-406.

7. K. Dabrowski, Moduli spaces for Hopf surfaces, Math. Ann. 299 (1982), 201-225.

8. A. Fröhlihcher and A. Nijenhuis, Theory of vector-valued forms I. Derivations in the graded ring of differential forms, Nedrl. Akad. Wetensch. Proc. Ser. A 59 (1956), 338-359.

9. P. Gauduchon, Hermitian connections and Dirac operators, Boll. Un. Mat. Ital B 11 (1997), 257-288.

10. W. M. Goldman and J. J. Milson, The homotopy invariance of the Kuranishi space, Illinois J. Math. 34 (1990), 337-367.

11. P. Griffiths, Some geometric and analytic properties of homogeneous complex manifolds, Acta Math. 110 (1963), 115-208.

12. F. Hirzebruch, Topological methods in algebraic geometry Third edition (SpringerVerlag, 1978).

13. K. Kodaira, On the structure of compact complex analytic surfaces, I. Amer. J. Math. 86 (1964), 751-798.

14. K. Kodaira and D. C. Spencer, On deformations of complex analytic structures, I, II, Ann. of Math. 68 (1958), 450-459.

15. M. Kuranishi. On the locally complete families of complex analytic structures, Ann. of Math. 75 (1962), 536-577.

16. S. Salamon. Complex structures on nilpotent Lie algebras, J. Pure Appl. Algebra 157 (2001), 311-333. 132.

17. H. Samelson. A class of complex-analytic manifolds, Portugal. Math. 12 (1953), 129

18. R. O. Wells, Jr. Differential analysis on complex manifolds (Springer-Verlag, 1979). 